NIST Technical Note 1984

\title{
The Influence of Walls, Corners and Enclosures on Fire Plumes
}

Kevin McGrattan

Michael Selepak

Edward Hnetkovsky
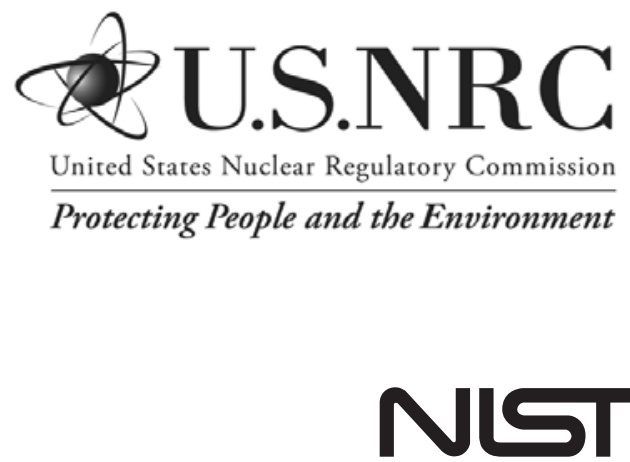

National Institute of Standards and Technology

U.S. Department of Commerce 
NIST Technical Note 1984

\title{
The Influence of Walls, Corners and Enclosures on Fire Plumes
}

\author{
Kevin McGrattan \\ Michael Selepak \\ Edward Hnetkovsky \\ Fire Research Division \\ Engineering Laboratory
}

This publication is available free of charge from:

https://doi.org/10.6028/NIST.TN.1984

March 2018
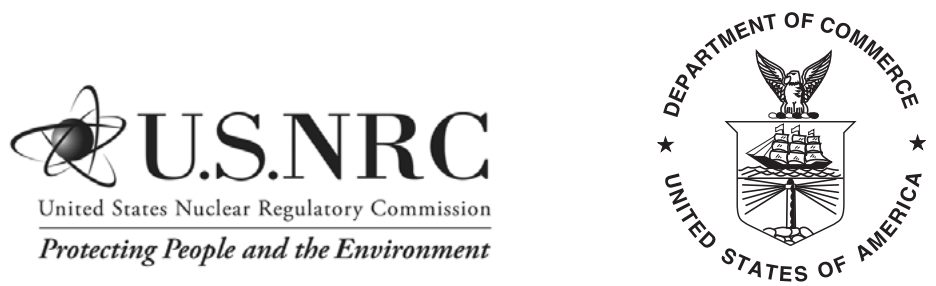

U.S. Department of Commerce Wilbur L. Ross, Jr., Secretary

National Institute of Standards and Technology Walter Copan, NIST Director and Undersecretary of Commerce for Standards and Technology 
Certain commercial entities, equipment, or materials may be identified in this document in order to describe an experimental procedure or concept adequately. Such identification is not intended to imply recommendation or endorsement by the National Institute of Standards and Technology, nor is it intended to imply that the entities, materials, or equipment are necessarily the best available for the purpose.

National Institute of Standards and Technology Technical Note 1984 Natl. Inst. Stand. Technol. Tech. Note 1984, 49 pages (March 2018) CODEN: NTNOEF

This publication is available free of charge from: https://doi.org/10.6028/NIST.TN.1984 


\begin{abstract}
This report documents two series of compartment fire experiments in which a natural gas burner is positioned in a corner, or against a wall, or inside a steel cabinet, to assess the effects on the plume and compartment temperatures. The measurements consist of one dimensional vertical thermocouple arrays to measure the hot gas layer temperature and height, and a three dimensional thermocouple array to measure the temperature of the fire plume as the burner is moved away from the corner or wall. The primary purpose of the experiments is to provide validation data for fire modeling analyses of these effects.
\end{abstract}

\title{
Key words
}

Compartment Fires; Corner Effects; Electrical Enclosures; Wall Effects. 


\section{Table of Contents}

1 Introduction $\quad 1$

1.1 Corner and Wall Effects 1

1.2 Enclosure Effects 1

1.3 Objective 2

2 Description of the Facility 3

2.1 Test Compartment 3

2.2 Burners 3

2.3 Instrumentation $\quad 6$

3 Wall and Corner Experiments $\quad 12$

3.1 Procedure $r$

3.2 Results 16

$\begin{array}{ll}\text { 3.2.1 Plume Temperatures } & 16\end{array}$

3.2.2 Hot Gas Layer Temperature and Height 19

4 Cabinet Experiments $\quad 22$

4.1 Description of Cabinets 22

4.2 Procedure 28

$\begin{array}{lll}4.3 & \text { Results } & 35\end{array}$

4.3.1 Cabinet Temperatures and Heat Flux 35

4.3.2 Ventilation Effects 37

4.3.3 Comparison with Experiments with no Cabinets 39

5 Conclusion $\quad 42$

Acknowledgments $\quad 42$

$\begin{array}{ll}\text { References } & 43\end{array}$

\section{List of Tables}

Table 1 Summary of Cabinet Experiments.

\section{List of Figures}

Fig. 1 Photograph of the exterior of the test compartment 4

Fig. 2 Photograph of the ganged burners used in the wall and corner experiments 5

Fig. 3 Photograph of the strip of mineral wool sealing the burner 5

Fig. 4 Photograph of the 3-D TC array 7

Fig. 5 Photograph of burner against the wall 8

Fig. 6 Close-up of a plate thermometer 9

Fig. 7 Diagram of thermocouple layout in the 3-D array 10

Fig. 8 Plan view of the compartment showing locations of the corner and wall burners 12 
Fig. 9 Photographs of $300 \mathrm{~kW}$ corner fire 14

Fig. 10 Photographs of $400 \mathrm{~kW}$ wall fire $\quad 15$

Fig. 11 Plume temperatures, 200, 300, $400 \mathrm{~kW}$ corner fires 17

Fig. 12 Plume temperatures, 200, 300, $400 \mathrm{~kW}$ wall fires 18

Fig. 13 HGL temperature and height, 200,300, $400 \mathrm{~kW}$ corner fires 20

Fig. 14 HGL temperature and height, 200, 300, $400 \mathrm{~kW}$ wall fires 21

Fig. 15 Large cabinet drawing, NIST/NRC Corner Effects Experiments 23

Fig. 16 Photograph of the large cabinet 24

Fig. 17 Medium-sized cabinet drawing, NIST/NRC Corner Effects Experiments 25

Fig. 18 Photograph of the medium-sized cabinet 26

Fig. 19 Cabinet grill, NIST/NRC Corner Effects Experiments 27

Fig. 20 Photograph of the grill over the large cabinet upper vent 27

Fig. 21 Plan view of the compartment showing locations of the cabinets 28

Fig. 22 Photograph of large cabinet with $400 \mathrm{~kW}$ fire 29

Fig. 23 Photograph of large cabinet with $1000 \mathrm{~kW}$ fire 30

Fig. 24 Photograph of medium cabinet with $200 \mathrm{~kW}$ fire 32

Fig. 25 Photograph of medium cabinet with $325 \mathrm{~kW}$ fire 32

Fig. 26 Plan view of the compartment, Cabinet Tests 11 and 12

Fig. 27 Configuration of Tests 11 and 12

Fig. 28 Photograph of Test 11, $1000 \mathrm{~kW} \quad 34$

Fig. 29 Gas and steel temperature of the large cabinet, Test 5

Fig. 30 Heat flux to nearby targets, Test 5

Fig. 31 Heat release rate, Tests 7 and $8 \quad 38$

Fig. 32 HGL temperature, Tests 7 and 8

Fig. 33 HGL temperature for large cabinet experiments where front door is closed 40

Fig. 34 HGL temperature for large cabinet experiments where front door is open $\quad 40$

Fig. 35 Plume temperatures for Tests 4 and 12 


\section{Introduction}

An early and important advance in fire science was the development of empirical correlations describing plume temperature, velocity, and flame height. These correlations were derived from experiments involving unobstructed fire plumes within relatively large, open compartments. However, in many hazard analyses, the fire is located in a corner, or against a wall, or inside a relatively small enclosure, like an electrical cabinet. This potentially changes the fire behavior and necessitates a modification of the correlations. The experiments described in this report are intended to validate these modifications.

\subsection{Corner and Wall Effects}

The centerline temperature rise, $\Delta T_{0}(z)$, of an unobstructed fire plume at a height $z$ above the base of a fire can be estimated using Heskestad's correlation [1]:

$$
\Delta T_{0}(z)=9.1\left(\frac{T_{\infty}}{g c_{p}^{2} \rho_{\infty}^{2}}\right)^{1 / 3} \dot{Q}_{\mathrm{c}}^{2 / 3}\left(z-z_{0}\right)^{-5 / 3}
$$

where $T_{\infty}$ is the ambient temperature, $g$ is the acceleration of gravity, $c_{p}$ is the specific heat of air, $\rho_{\infty}$ is the ambient air density, $\dot{Q}_{\mathrm{c}}$ is the convective heat release rate, and $z_{0}$ is the "virtual" origin, given by

$$
\frac{z_{0}}{D}=-1.02+1.4 \dot{Q}^{* 2 / 5} \quad ; \quad \dot{Q}^{*}=\frac{\dot{Q}}{\rho_{\infty} c_{p} T_{\infty} \sqrt{g D} D^{2}}
$$

Here, $D$ is the diameter of the fire base. Heskestad provides references to studies that consider the effects of walls and corners on the fire plume. A simple modification to Eq. 1 is to multiply the heat release rate and the fire's base area by a factor of 2 or 4, respectively, to account for the effect of a wall or corner. The idea is that the temperature and entrainment rate of these fires can be estimated by evaluating their mirror images in Eq. 1. One of the goals of the experiments described in this report is to provide empirical evidence to support or refute this simple modification.

\subsection{Enclosure Effects}

In 2013-2014, NIST conducted 112 experiments to measure the heat release rate (HRR) of electrical enclosures with a variety of combustible loadings, ventilation conditions, geometries, and ignition sources [2]. Following these experiments, a working group was convened by the U.S. Nuclear Regulatory Commission to determine appropriate statistical distributions for the peak HRR of these and previously conducted electrical enclosure fire experiments [3].

However, an appropriate HRR for a given type of electrical enclosure is a necessary but not sufficient piece of information for the fire modeling analysis. The other important 
piece is the role played by the enclosure itself in changing the basic fire dynamics. Simpler fire models, including empirical correlations and zone models, do not include an explicit algorithm to account for the effects of the enclosure. These models assume that the fire plume is free of all obstructions, i.e. axisymmetric, except for the compartment ceiling. Obviously, a steel enclosure surrounding the fire is going to disrupt the plume and absorb some of the heat, depending on its geometry. This will result in less heat transported to the hot gas layer (HGL) above the electrical enclosure, and potentially lower target and HGL temperatures. A computational fluid dynamics (CFD) model can simulate a fire within a steel enclosure, and such modeling has been performed in Ref. [3] to estimate the enclosure's effect on the plume temperature. However, to date, CFD fire models have not been validated for this fairly specific application. The experiments described in this report were designed to provide data for this purpose.

\subsection{Objective}

The objective of the experiments described in this report is to provide measurement data with which to validate fire models used in industrial design applications.

With regard to wall and corner fires, while there have been a number of experimental studies [1] to examine the effect on plume temperature and height, there is little data that indicates how much the hot gas layer temperature is affected by the corner and wall effects. In addition, there is limited guidance on the necessary distance away from the wall or corner required for the fire to no longer be significantly affected. In other words, what constitutes a fire being "in the corner" or "against a wall".

With regard to enclosure effects, it is difficult to develop a simple modification to plume temperature and entrainment correlations because there is a limitless number of enclosure geometries and materials. It is hoped that current generation computational fluid dynamics (CFD) models can be applied to specific configurations in lieu of expensive full-scale experiments, but such models first must be validated against experiments that are of comparable scope. 


\section{Description of the Facility}

In the summer of 2017, experiments were conducted at the National Fire Research Laboratory at NIST, Gaithersburg, on behalf of the U.S. Nuclear Regulatory Commission. There were two sets of experiments, both conducted in the same large test compartment. In the first set, conducted in July, 2017, a natural gas burner was positioned either in a corner or against a wall and gradually moved outward to assess the effect of the wall or corner on the fire behavior. In the second set of experiments, conducted in September, 2017, a natural gas burner was placed inside one of two steel cabinets meant to represent typical industrial-scale electrical enclosures. The heat release rate was varied to mimic those of fires expected in such enclosures.

This chapter describes the compartment and instrumentation used in both sets of experiments.

\subsection{Test Compartment}

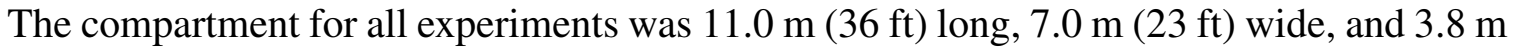
$(12.5 \mathrm{ft})$ high. The long dimension of the compartment ran east-west. A $1.8 \mathrm{~m}(6 \mathrm{ft})$ wide, $2.4 \mathrm{~m}(8 \mathrm{ft})$ high door was centered on the east (short) wall. The compartment walls and ceiling were lined with $13 \mathrm{~mm}(0.5 \mathrm{in})$ thick gypsum board ${ }^{1}$, and the floor was covered with $13 \mathrm{~mm}(0.5 \mathrm{in})$ thick plywood covered by the same gypsum board panels that lined the walls and ceiling. An extra layer of $6 \mathrm{~mm}\left(0.25\right.$ in) thick cement board ${ }^{2}$ was added to the walls and ceiling of the southwest quarter of the compartment, extending $7 \mathrm{~m} \mathrm{(23 \textrm {ft } )}$ along the south wall and $3 \mathrm{~m}(10 \mathrm{ft})$ along the west wall, with corresponding coverage on the ceiling.

\subsection{Burners}

All of the fires were fueled by one or more $30.5 \mathrm{~cm}(1 \mathrm{ft})$ square natural gas burners. Each burner was essentially a steel box, $30.5 \mathrm{~cm}$ (1 ft) square in plan and $15 \mathrm{~cm}$ (6 in) deep, fueled from below. The lip of the burner was $2.5 \mathrm{~cm}$ (1 in) wide. A $2.5 \mathrm{~cm}$ (1 in) thick piece of mineral wool was placed under a steel mesh to form the surface of the burner.

The heat release rates (HRR) for all experiments ranged from $25 \mathrm{~kW}$ to $1000 \mathrm{~kW}$. For the experiments with peak HRR less than $500 \mathrm{~kW}$, a mass flow controller ${ }^{3}$ was used to measure the mass flow rate of natural gas to the burner(s). For experiments with peak HRR greater than $500 \mathrm{~kW}$, a positive displacement flow meter ${ }^{4}$ was used. Internal calibrations at

${ }^{1}$ U.S. Gypsum Sheetrock brand gypsum panels. Estimated thermal properties from product literature are: specific heat, $1.1 \mathrm{~kJ} /(\mathrm{kg} \cdot \mathrm{K})$, density, $700 \mathrm{~kg} / \mathrm{m}^{3}$, and thermal conductivity, $0.16 \mathrm{~W} /(\mathrm{m} \cdot \mathrm{K})$.

${ }^{2}$ U.S. Gypsum Durock brand cement board. Estimated thermal properties from product literature are: specific heat, $1.0 \mathrm{~kJ} /(\mathrm{kg} \cdot \mathrm{K})$, density, $925 \mathrm{~kg} / \mathrm{m}^{3}$, and thermal conductivity, $0.15 \mathrm{~W} /(\mathrm{m} \cdot \mathrm{K})$.

${ }^{3}$ Alicat Scientific, Inc., Tucson, Arizona, Model MCR-1000SLPM-D.

${ }^{4}$ Elster Instromet, Inc., Houston, Texas, Model IRM-3 DUO SIZE 38 M. 


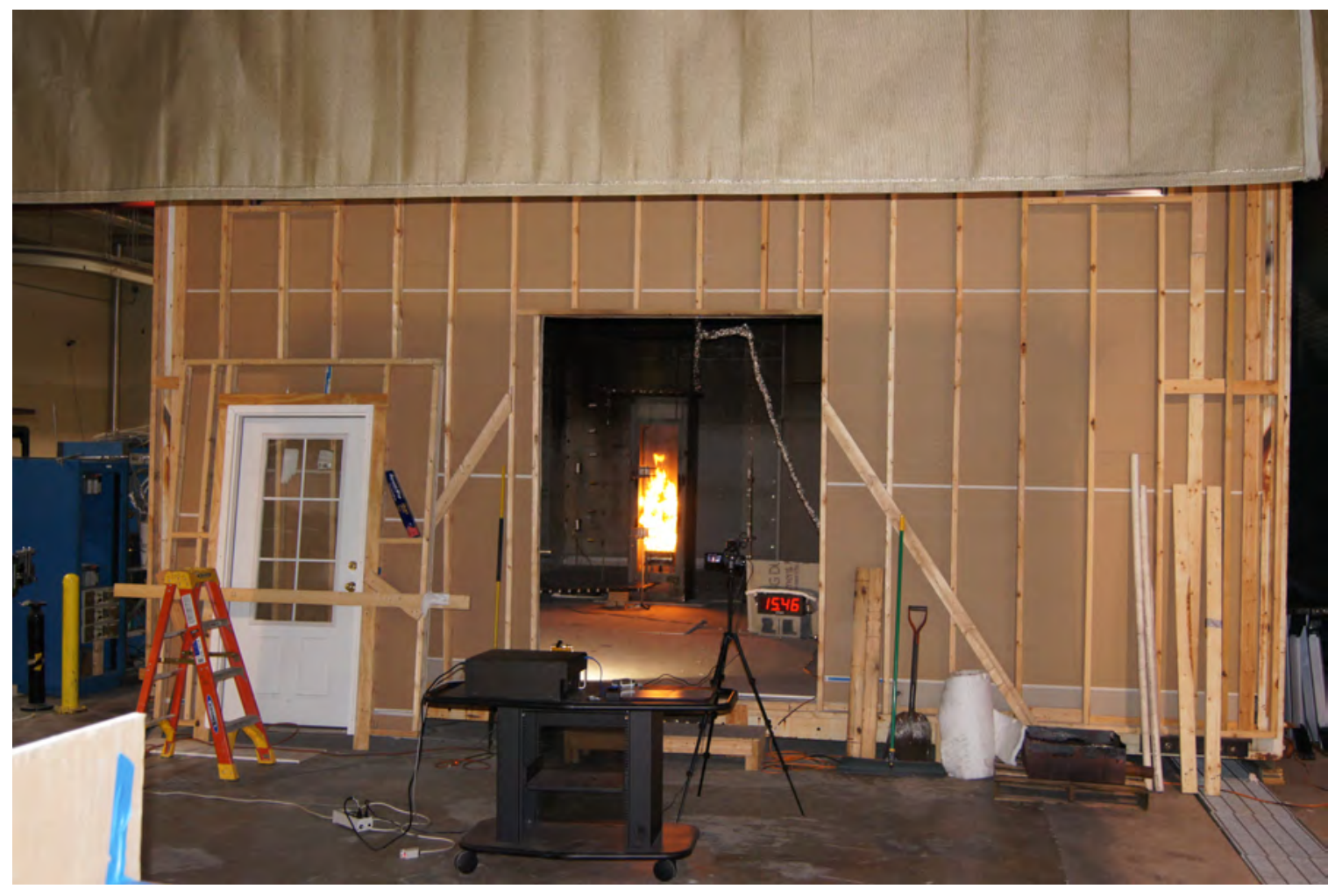

Fig. 1. Photograph of the exterior of the test compartment, looking through the opening on the east wall. The south wall is to the left; north to the right; and west in the background.

NIST indicate that both devices have an expanded ${ }^{5}$ relative uncertainty of approximately $1 \%$ for the range of flow rates required.

In the wall and corner experiments, four burners were ganged together and mounted on steel rails as shown in Fig. 2. The steel rails allowed the burners to be pushed outward from the corner and wall during the experiments. The burner was not shut off during the moves. To ensure that the burner was initially tight against the wall and corner, a strip of mineral wool was inserted to seal the gap, as shown in Fig. 3. The quad burner was $60 \mathrm{~cm}$ (24 in) by $60 \mathrm{~cm}$ ( $24 \mathrm{in})$ and the burner surface was $54 \mathrm{~cm}$ (21 in) above the floor. The corner fire was located in the southwest corner of the large compartment. The wall fire was centered on the south (long) wall.

For the cabinet experiments, two $30.5 \mathrm{~cm}$ (12 in) burners were arranged side by side and centered within the larger of the two cabinets. The fuel pipe entered through the slot at the bottom front. For the smaller cabinet, a single $30.5 \mathrm{~cm}$ (12 in) burner was placed inside, centered. The upper surface of the cabinet burners was $50 \mathrm{~cm}$ (20 in) above the floor.

$595 \%$ confidence interval 


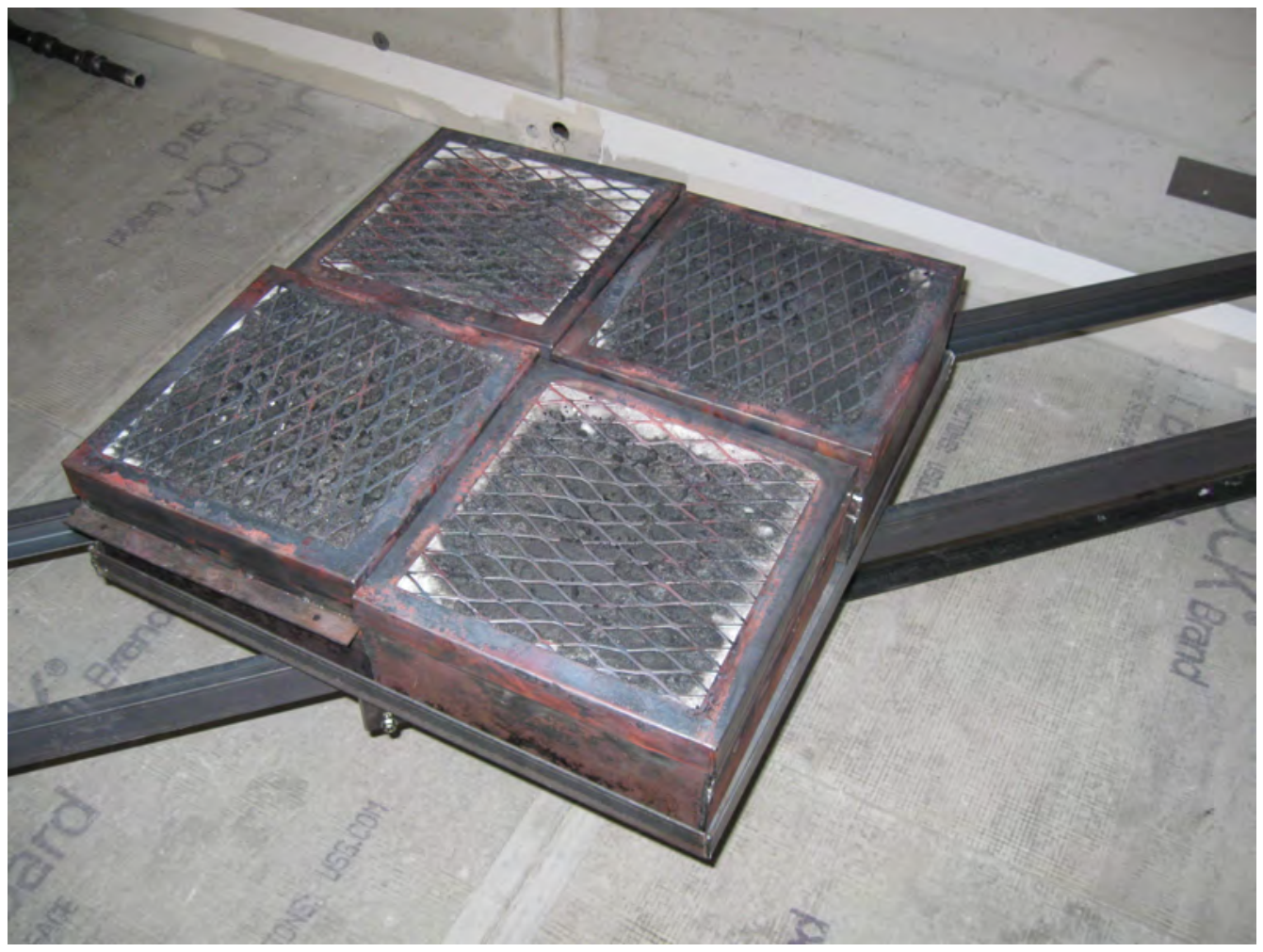

Fig. 2. Photograph of the ganged burners used in the wall and corner experiments.

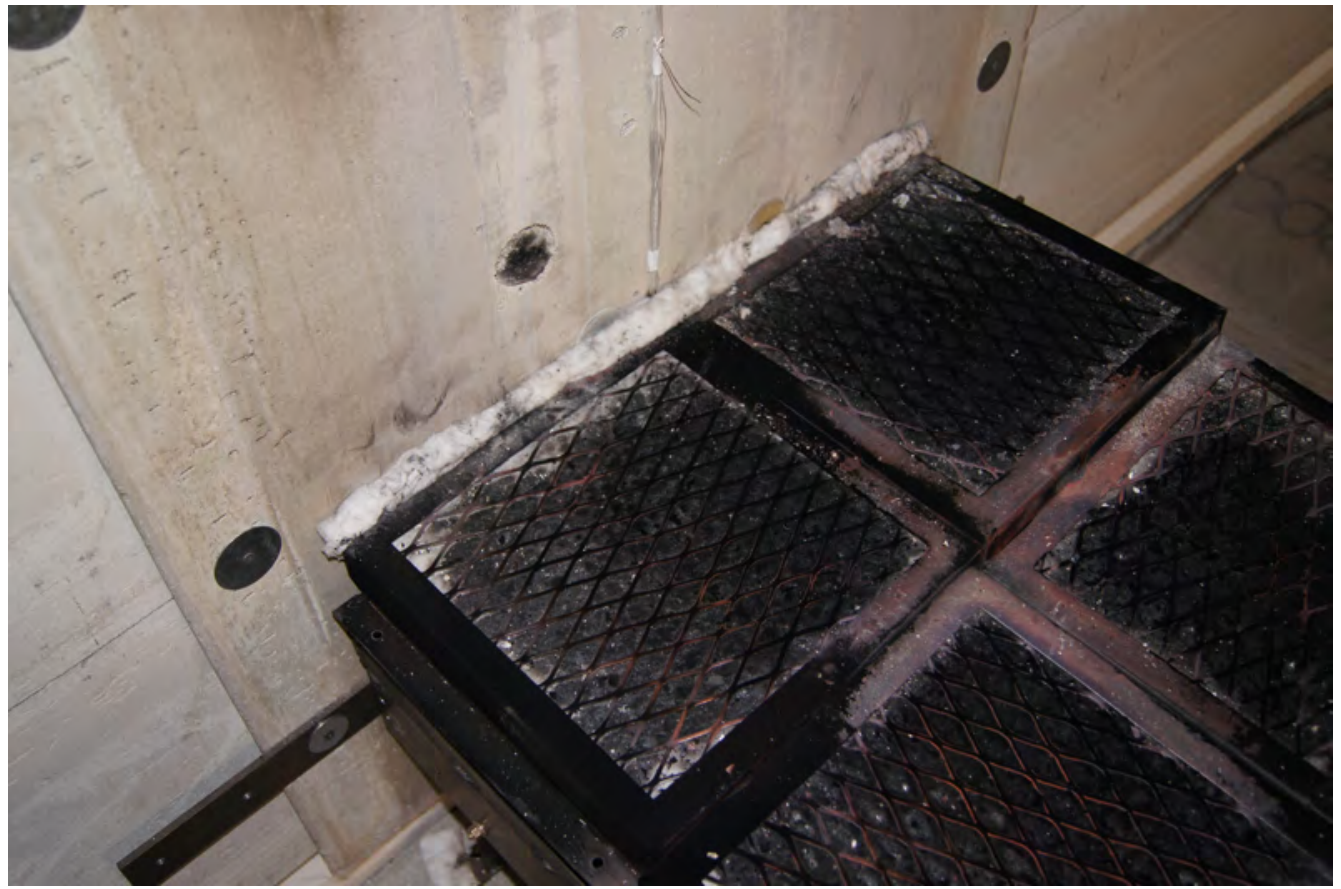

Fig. 3. Photograph of the strip of mineral wool sealing the gap. 


\subsection{Instrumentation}

The primary measurement device was a 30 gauge $^{6}$ Type $\mathrm{K}$ thermocouple (TC). The stated accuracy, the so-called "Special Limits of Error," is $\pm 1.1^{\circ} \mathrm{C}$ or $\pm 0.4 \%$ of the temperature expressed in ${ }^{\circ} \mathrm{C}$, whichever is greater. However, the much greater source of uncertainty of bare-bead thermocouple measurements is thermal radiation from distant heat sources. For example, thermocouples in the relatively cool lower layer of the fire compartment record higher temperatures because they sense the thermal radiation from the fire and the relatively hot upper layer. It is not possible to succinctly state the true uncertainty of each thermocouple, but modelers are advised to predict the temperature of an approximately $0.5 \mathrm{~mm}$ (0.02 in) diameter metal sphere rather than the actual gas temperature [4]. The bare-bead TC measurements reported here have not been corrected in any way.

There were four vertical TC arrays spanning the height of the compartment. One was located approximately $2 \mathrm{~cm}$ ( 1 in) from the southwest corner of the compartment where the corner fire experiments were conducted. A similar array was mounted halfway along the south wall where the wall fire experiments were conducted. Two arrays were located along the compartment centerline, at distances of one-third and two-thirds of the compartment length from the open door. These arrays were used to calculate the hot gas layer temperature and height.

A three-dimensional array of thermocouples was mounted to rails attached to the ceiling above the burner (see Figs. 4 and 5). The array consisted of three $91 \mathrm{~cm}$ by $91 \mathrm{~cm} \mathrm{(36} \mathrm{in} \mathrm{by}$ 36 in) steel grids connected via vertical struts to a frame suspended below the ceiling. The TC beads extended $5 \mathrm{~cm}$ ( 2 in) below the grid. The array travelled with the moveable burner

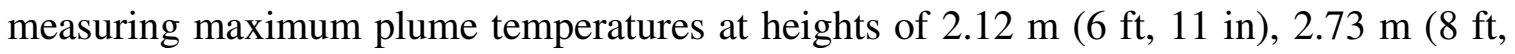
$11 \mathrm{in})$, and $3.34 \mathrm{~m} \mathrm{(10} \mathrm{ft,} 11 \mathrm{in})$ above the floor. For the corner and wall fire experiments, when the burner was at the $0 \mathrm{~cm}, 10 \mathrm{~cm}$ (4 in), and $20 \mathrm{~cm}(8 \mathrm{in})$ positions, the thermocouple array overhead remained at its original location in the corner or against the wall. As the burner moved beyond $20 \mathrm{~cm}$ (8 in), the thermocouple array was moved the same amount so that the burner was always below the array in the same position. In other words, after the center point of the burner reached the point directly below the position 18 on the diagram in Fig. 7, the burner and array moved together, maintaining their relative position. For the cabinet experiments, the 3-D array was fixed just above the front of the cabinet to measure the temperature of the hot gases exiting the front door and vent. The TCs at positions 1, 7, 13, 19, and 25 in Fig. 7 were just above the upper front edge of the cabinet.

For the cabinet experiments, plate thermometers were positioned at a lateral distance of

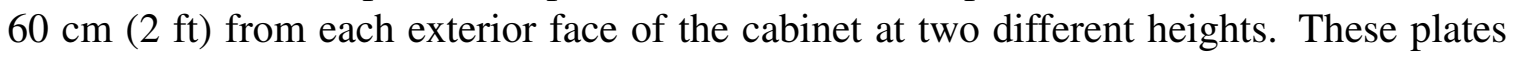
were made at NIST (see Fig. 6). The heat flux to the plate thermometer can be calculated from its measured temperature using the heat balance equation suggested by Ingason and Wickström [5]:

$$
\dot{q}^{\prime \prime}=\sigma\left(T_{\mathrm{PT}}^{4}-T_{\infty}^{4}\right)+\frac{\left(h_{\mathrm{PT}}+K_{\mathrm{con}}\right)\left(T_{\mathrm{PT}}-T_{\mathrm{gas}}\right)}{\varepsilon_{\mathrm{PT}}}+\frac{\rho_{\mathrm{PT}} c_{\mathrm{PT}} \delta\left(\Delta T_{\mathrm{PT}} / \Delta t\right)}{\varepsilon_{\mathrm{PT}}}
$$

630 gauge wire is $0.254 \mathrm{~mm}$ in diameter. 


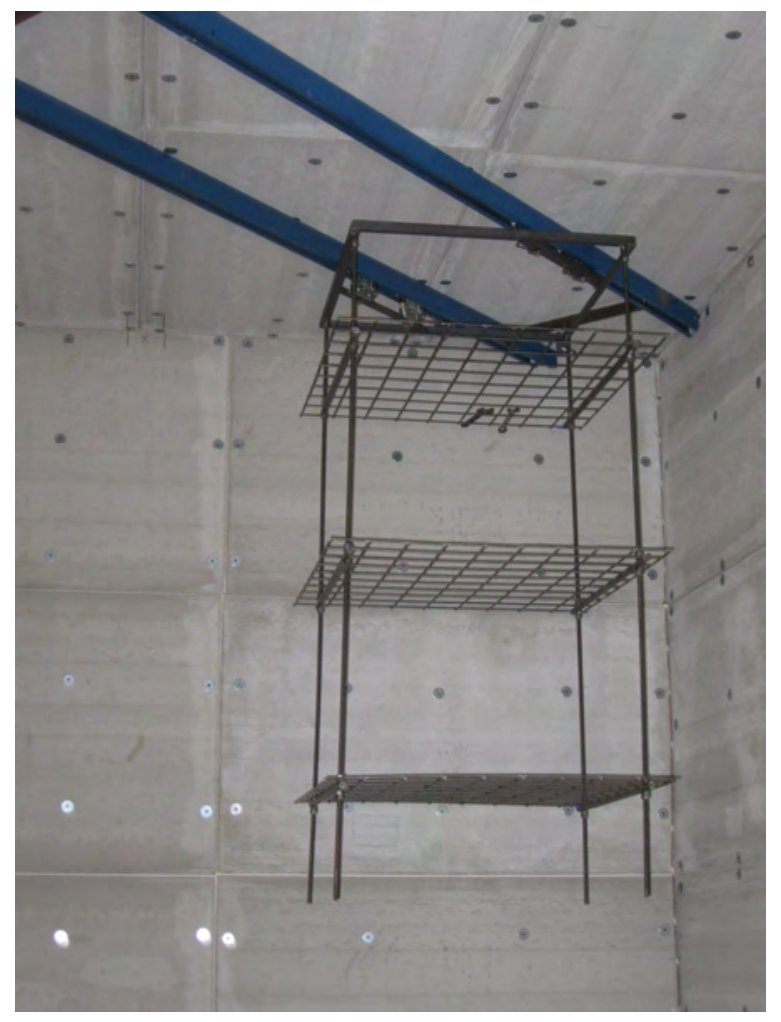

Fig. 4. Photograph of the 3-D TC array.

Here $\dot{q}^{\prime \prime}$ is the "gauge" heat flux; that is, the heat flux one would measure with a watercooled gauge. $\sigma$ is the Stefan-Boltzmann constant, $5.67 \times 10^{8} \mathrm{~W} /\left(\mathrm{m}^{2} \cdot \mathrm{K}^{4}\right), T_{\mathrm{PT}}$ is the measured temperature of the plate $(\mathrm{K}), T_{\infty}$ is the ambient temperature $(\mathrm{K}), h_{\mathrm{PT}}$ is the assumed convective heat transfer coefficient, $10 \mathrm{~W} /\left(\mathrm{m}^{2} \cdot \mathrm{K}\right), K_{\text {con }}$ is an empirical conduction factor, $4 \mathrm{~W} /\left(\mathrm{m}^{2} \cdot \mathrm{K}\right), T_{\text {gas }}$ is the gas temperature in the vicinity of the plate $(\mathrm{K}), \varepsilon_{\mathrm{PT}}$ is the plate emissivity, $0.85, \rho_{\mathrm{PT}}$ is the plate density, $8470 \mathrm{~kg} / \mathrm{m}^{3}, c_{\mathrm{PT}}$ is the plate heat capacity, $502 \mathrm{~J} /(\mathrm{kg} \cdot \mathrm{K})$, $\delta$ is the plate thickness, $7.9 \times 10^{-4} \mathrm{~m}$, and $\Delta T_{\mathrm{PT}}$ is the change in plate temperature $(\mathrm{K})$ over the time increment of the measurements, $\Delta t(\mathrm{~s})$. The gas temperature near the plate, $T_{\text {gas }}$, is taken as the average of the two thermocouples located in the vertical arrays along the compartment centerline that are closest in height to the upper and lower plates.

Putorti et al. [6] estimate that the combined relative uncertainty of the heat flux derived from the plate thermometer measurement is $2.5 \%$, based on experiments conducted in a cone calorimeter set to $75 \mathrm{~kW} / \mathrm{m}^{2}$.

For each experiment, the temperature data are stored in a single comma-delimited (csv) file labelled:

NIST_NRC_Corner(Wall)_NOO_kW.csv (N=2, 3, 4)

for the corner and wall experiments, and

NIST_NRC_Cabinet_Test_N.csv ( $\mathrm{N}=1--12)$

for the cabinet experiments. The key to the column names within these files are as follows: 


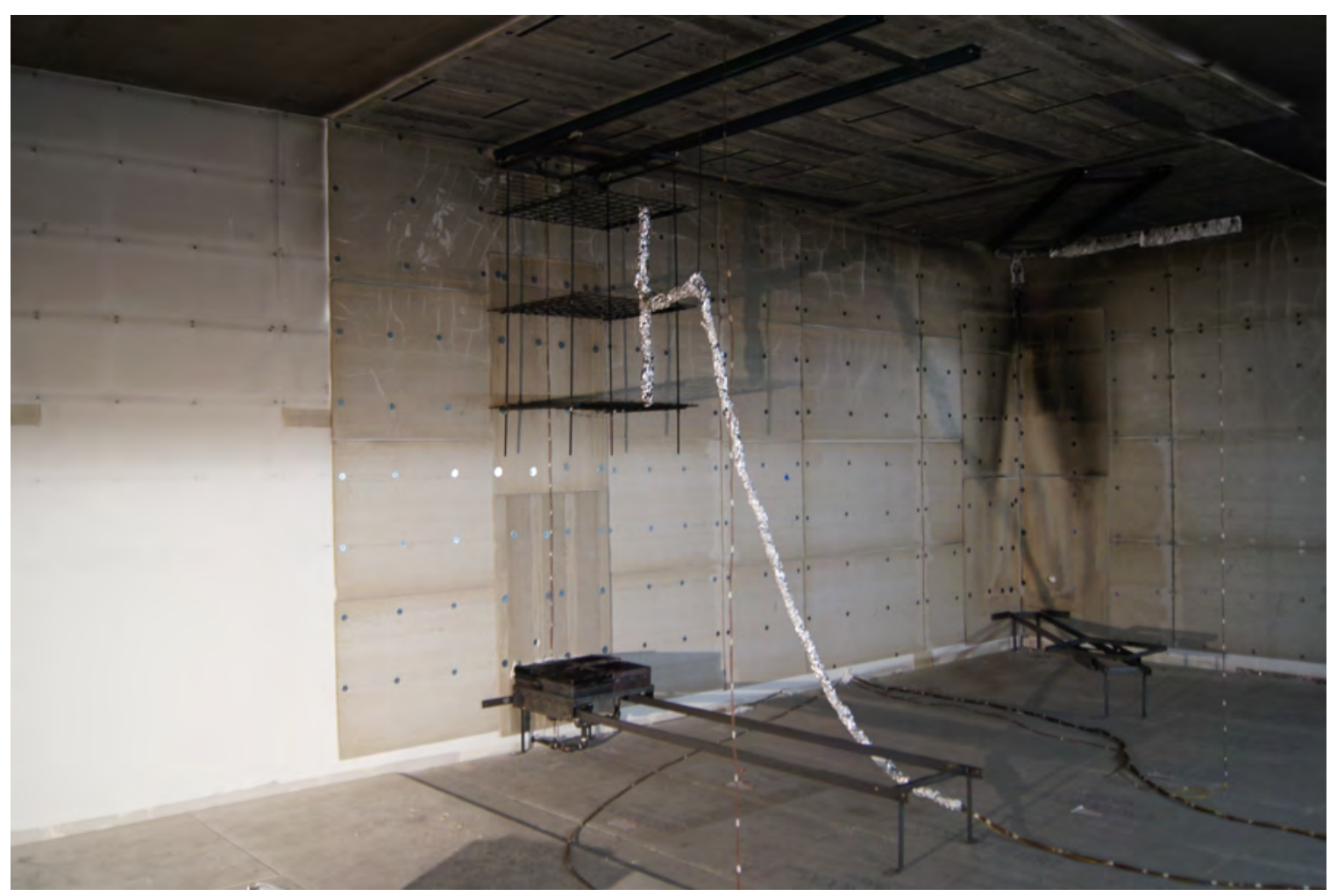

Fig. 5. Photograph of the wall fire experiment.

- TC-AG-01 through TC-AG-29 are the thermocouples at the top of the 3-D array, $46 \mathrm{~cm}$ (18 in) below the ceiling (see pattern below).

- TC-BG-01 through TC-BG-29 are the thermocouples at the mid-level of the 3-D array, $107 \mathrm{~cm}$ (42 in) below the ceiling (see pattern below).

- TC-CG-01 through TC-CG-29 are the thermocouples at the bottom of the 3-D array, $168 \mathrm{~cm}$ (66 in) below the ceiling (see pattern below).

- TC-WT-01 through TC-WT-13 are the thermocouples of the vertical array called the West Tree. The array was $2.75 \mathrm{~m}(9 \mathrm{ft})$ from the west (short) wall and $3.50 \mathrm{~m}(11 \mathrm{ft}$, 6 in) from the south (long) wall. TC-WT-01 was located $2 \mathrm{~cm}$ below the ceiling, and the rest were spaced $30 \mathrm{~cm}(1 \mathrm{ft})$ apart.

- TC-ET-01 through TC-ET-13 are the thermocouples of the East Tree. The array was

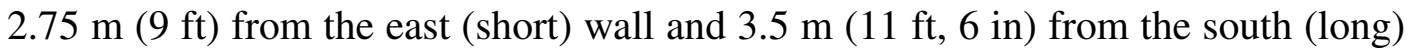
wall. TC-ET-01 was located $2 \mathrm{~cm}$ ( 1 in) below the ceiling, and the rest were spaced $30 \mathrm{~cm}(1 \mathrm{ft})$ apart.

- TC-C-01 through TC-C-11 are the thermocouples $2 \mathrm{~cm}$ (1 in) from the corner above the corner fire. TC-C-01 was located $2 \mathrm{~cm}$ (1 in) below the ceiling, and the rest were 


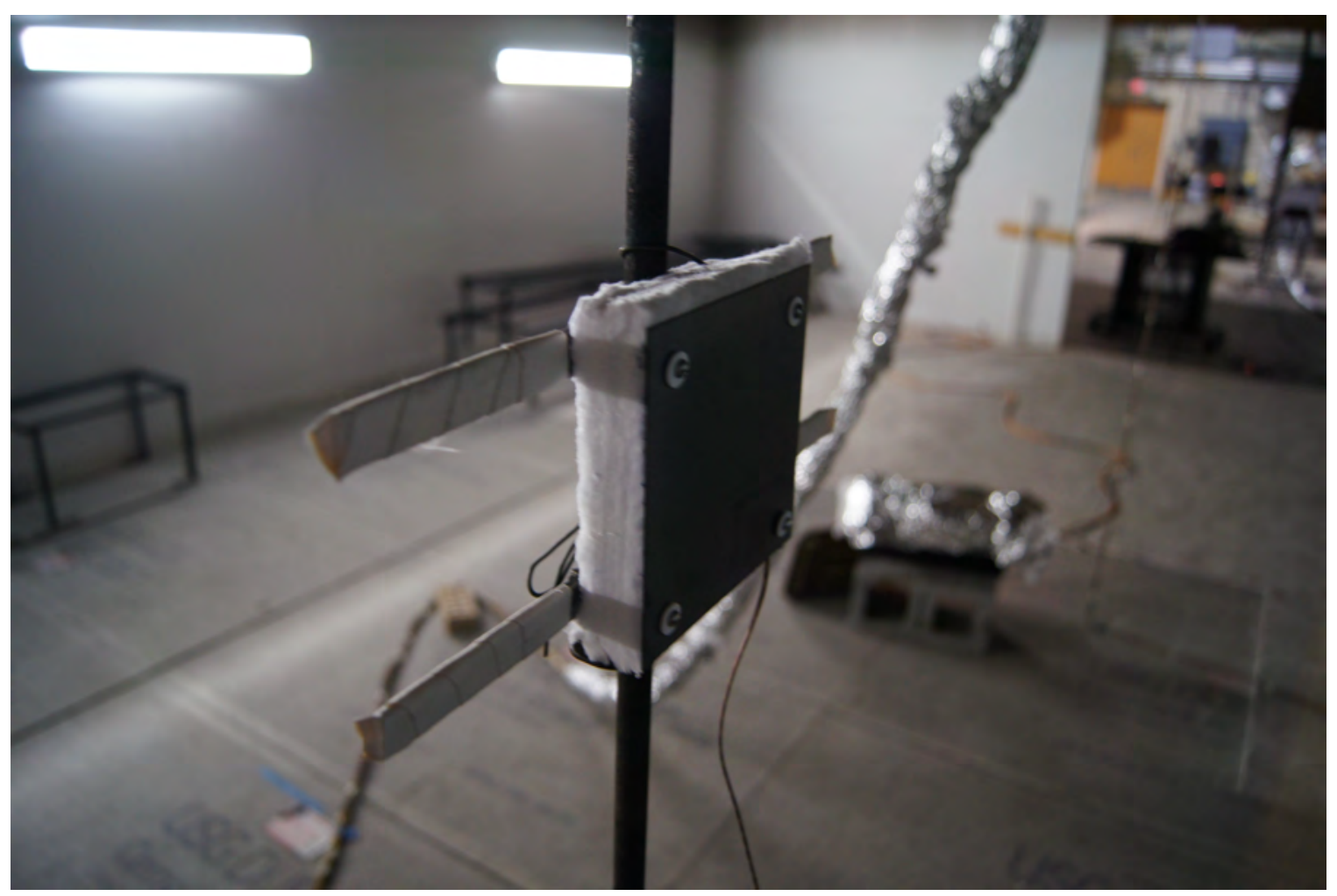

Fig. 6. Close-up of a plate thermometer.

spaced $30 \mathrm{~cm}(1 \mathrm{ft})$ apart.

- TC-W-01 through TC-W-11 are the thermocouples $2 \mathrm{~cm}$ (1 in) from the wall above the wall fire. TC-W-01 was located $2 \mathrm{~cm}$ (1 in) below the ceiling, and the rest were spaced $30 \mathrm{~cm}(1 \mathrm{ft})$ apart.

- HRR (cal) is the heat release rate of the fire as measured using oxygen consumption calorimetry. HRR (NG) is the heat release rate determined from the mass flow rate of natural gas.

For the cabinet effect experiments, there are the following additional measurements:

- PT-1 through PT-8 are plate thermometers positioned $0.6 \mathrm{~m}(2 \mathrm{ft})$ from each side of the cabinet at heights of $0.8 \mathrm{~m}(2.5 \mathrm{ft})$ and $1.4 \mathrm{~m}(4.5 \mathrm{ft})$. PT- 1 is the upper plate on the left side. PT-2 is lower left. PT-3 is upper back. PT-4 is lower back. PT-5 is upper front. PT-6 is lower front. PT-7 is upper right. PT-8 is lower right.

- STC-1 through STC-6 are sheathed thermocouples within the cabinet, $15 \mathrm{~cm}$ (6 in) from the left side, centered. STC-1 is $6 \mathrm{~cm}$ (2.5 in) from the top. STC-2 through STC-6 are $30 \mathrm{~cm}(1 \mathrm{ft}), 60 \mathrm{~cm}(2 \mathrm{ft}), 90 \mathrm{~cm}(3 \mathrm{ft}), 120 \mathrm{~cm}(4 \mathrm{ft})$, and $150 \mathrm{~cm}(5 \mathrm{ft})$ from the top, respectively. 
- TC-Cab is a single 24 gauge Type K thermocouple welded to the center of the back side on the outside of the cabinet. For Test 11, this TC was placed just a few millimeters below the top surface of the burner, and for Test 12, it was placed just above the surface.

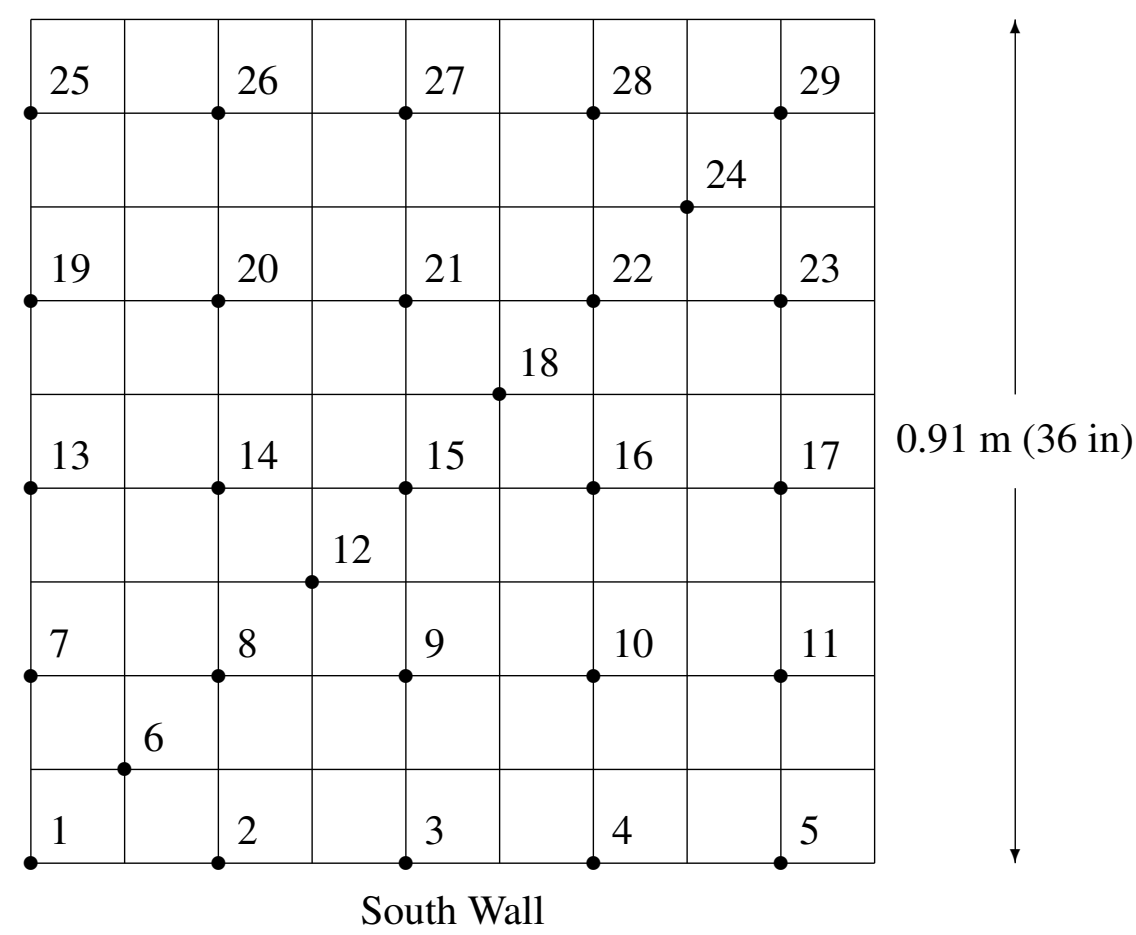

Fig. 7. Diagram of thermocouple layout in the 3-D array.

The East and West Tree thermocouples were used to estimate the height off the floor of the lower boundary of the hot gas layer (HGL), and the average temperatures of the upper and lower layers. Fire protection engineers often need to estimate the location of the interface between the hot, smoke-laden upper layer and the cooler lower layer in a burning compartment. One such method [7] is as follows: Consider a continuous function $T(z)$ defining temperature $T$ as a function of height above the floor $z$, where $z=0$ is the floor and $z=H$ is the ceiling. Define $T_{\mathrm{u}}$ as the upper layer temperature, $T_{\ell}$ as the lower layer temperature, and $z_{\text {int }}$ as the interface height. Compute the quantities:

$$
\begin{gathered}
\left(H-z_{\text {int }}\right) T_{\mathrm{u}}+z_{\text {int }} T_{\ell}=\int_{0}^{H} T(z) d z=I_{1} \\
\left(H-z_{\text {int }}\right) \frac{1}{T_{\mathrm{u}}}+z_{\text {int }} \frac{1}{T_{\ell}}=\int_{0}^{H} \frac{1}{T(z)} d z=I_{2}
\end{gathered}
$$

Solve for $z_{\text {int }}$ :

$$
z_{\text {int }}=\frac{T_{\ell}\left(I_{1} I_{2}-H^{2}\right)}{I_{1}+I_{2} T_{\ell}^{2}-2 T_{\ell} H}
$$


Let $T_{\ell}$ be the temperature in the lowest mesh cell and, using Simpson's Rule, perform the numerical integration of $I_{1}$ and $I_{2} . T_{\mathrm{u}}$ is defined as the average upper layer temperature via

$$
\left(H-z_{\text {int }}\right) T_{\mathrm{u}}=\int_{z_{\text {int }}}^{H} T(z) d z
$$

Further discussion of similar procedures can be found in Ref. [8].

The three horizontal arrays of thermocouples above the burner were processed by first taking a 2 min running average of each TC, and then choosing the maximum value for each of the three elevations above the fire. These were taken as approximate centerline plume temperatures at each height. These experimental files have the same name as the primary data file, but with the suffix "HGL" and "Plume" appended. 


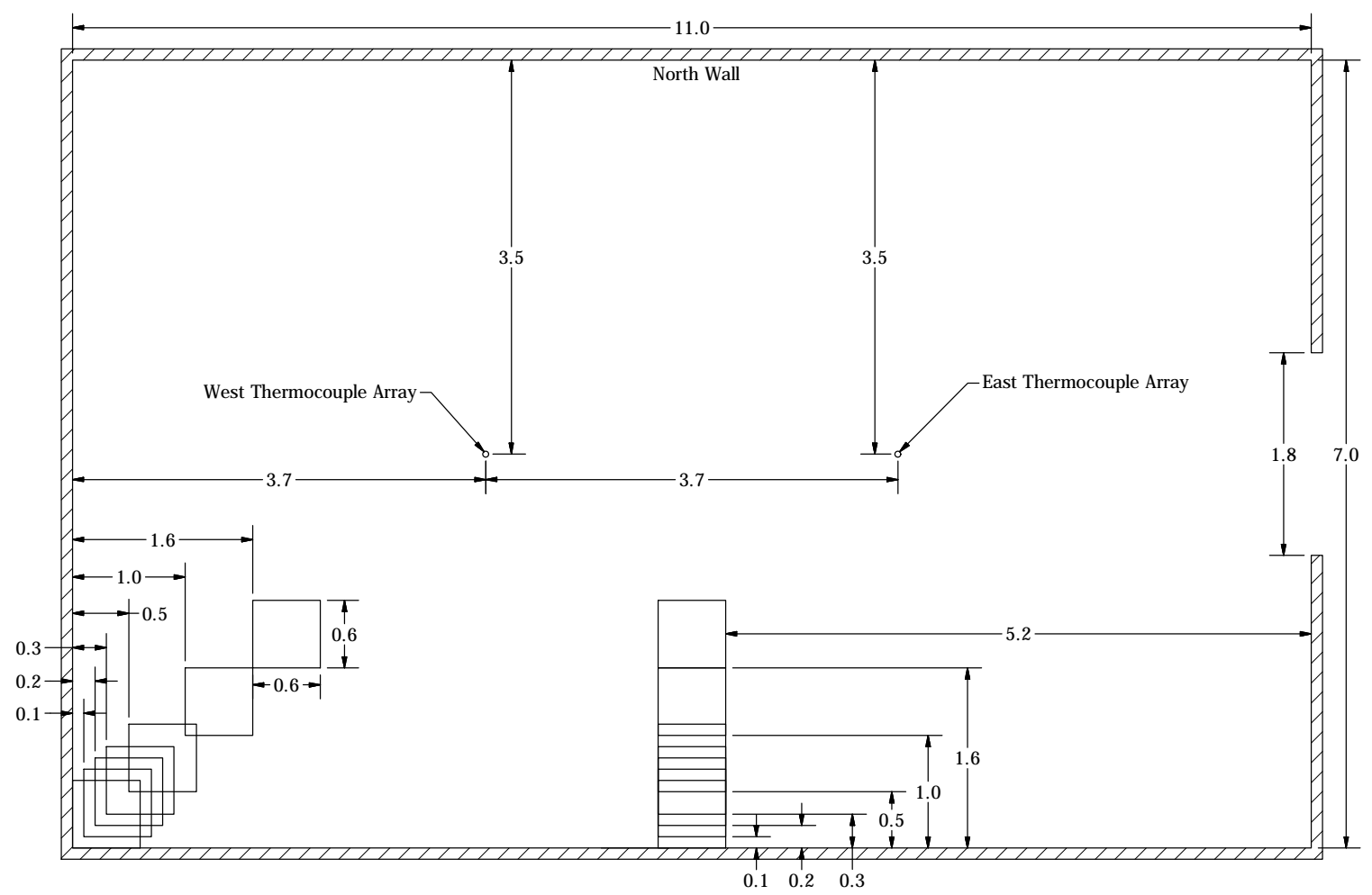

Fig. 8. Plan view of the compartment showing locations of the corner and wall burners. All dimensions are in meters.

\section{Wall and Corner Experiments}

Six large compartment experiments were conducted in July, 2017, where four natural gas burners were positioned (1) in a corner and (2) against a wall, and then moved outward in stages until the corner or wall effect became negligible.

\subsection{Procedure}

Experiments were conducted with fires of $200 \mathrm{~kW}, 300 \mathrm{~kW}$, and $400 \mathrm{~kW}$ for both the wall and corner configuration. The gas flow rate was ramped up to the desired value within approximately $10 \mathrm{~s}$ following ignition from a small propane torch. The gas flow was held steady for $2 \mathrm{~h}$ as the burner was moved from position to position. The experiments began with the quad burner in the corner or against the wall for the first $30 \mathrm{~min}$. At $30 \mathrm{~min}$, the burner was moved so that its edge(s) was $10 \mathrm{~cm}$ (4 in) away from the wall(s). It remained for $15 \mathrm{~min}$, after which it was moved to $20 \mathrm{~cm}(8 \mathrm{in}), 30 \mathrm{~cm}(12 \mathrm{in}), 50 \mathrm{~cm}(20 \mathrm{in}), 100 \mathrm{~cm}$ (40 in), and $160 \mathrm{~cm}$ (63 in), each time remaining $15 \mathrm{~min}$ for a total experiment time of $2 \mathrm{~h}$. The layout of the compartment is shown in Fig. 8.

Figure 9 shows a sequence of photographs of the $300 \mathrm{~kW}$ corner fire, and Fig. 10 shows photographs of the $400 \mathrm{~kW}$ wall fire. Although it is not readily apparent from the 
photographs, the corner fire flame heights decreased noticeably after the opening of the initial $10 \mathrm{~cm}$ gap. Subsequent shifts did not lead to noticeable changes in flame height, although the plume temperatures at the three measurement locations above the fire did continue to decrease. The wall fires exhibited no obvious change in flame height, nor a significant change in plume temperatures. 

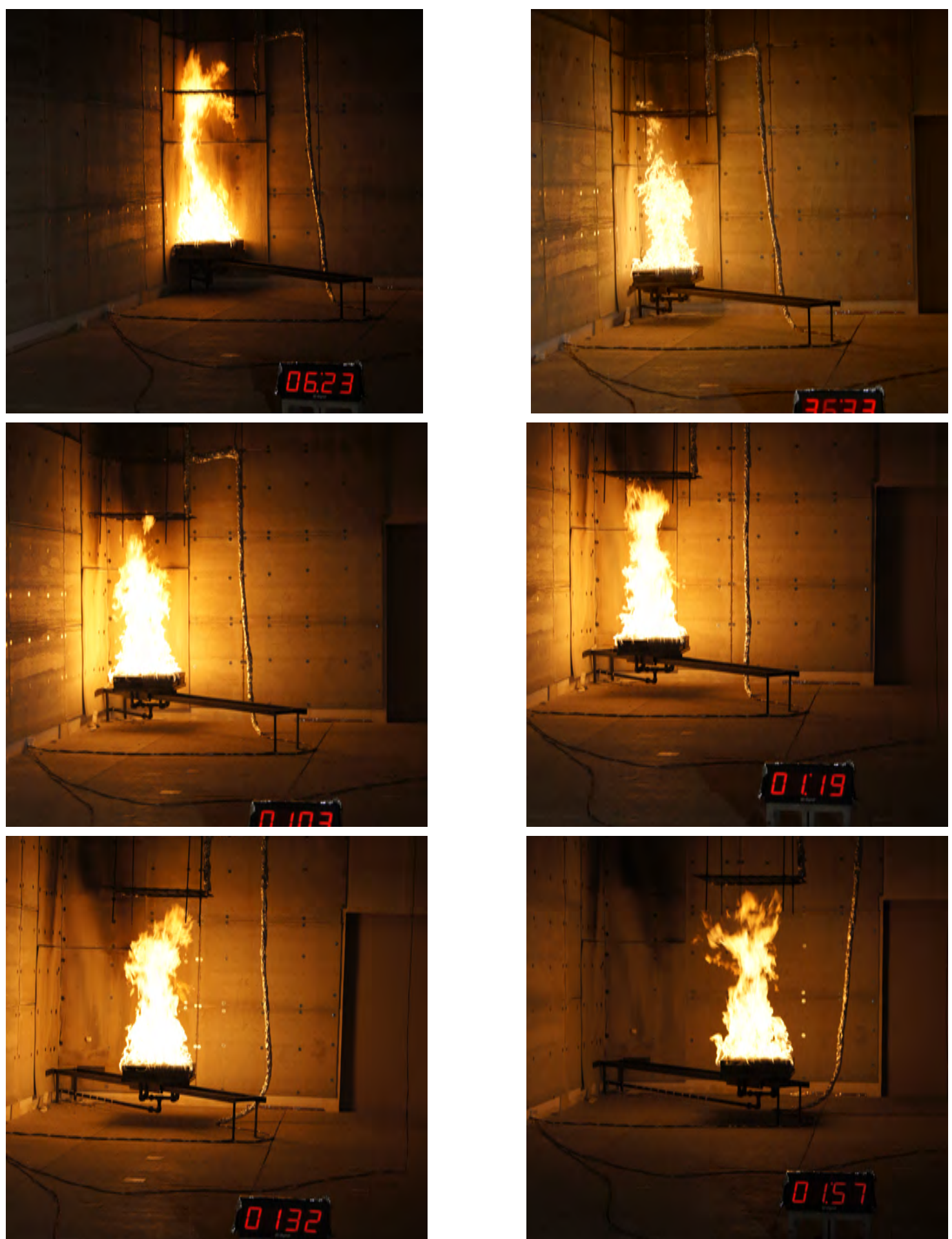

Fig. 9. Photographs of $300 \mathrm{~kW}$ corner fire at distances from the corner of $0 \mathrm{~cm}, 10 \mathrm{~cm}(4 \mathrm{in})$, $30 \mathrm{~cm}$ (12 in), $50 \mathrm{~cm}$ (20 in), $100 \mathrm{~cm}$ (40 in), and $160 \mathrm{~cm}$ (63 in). 

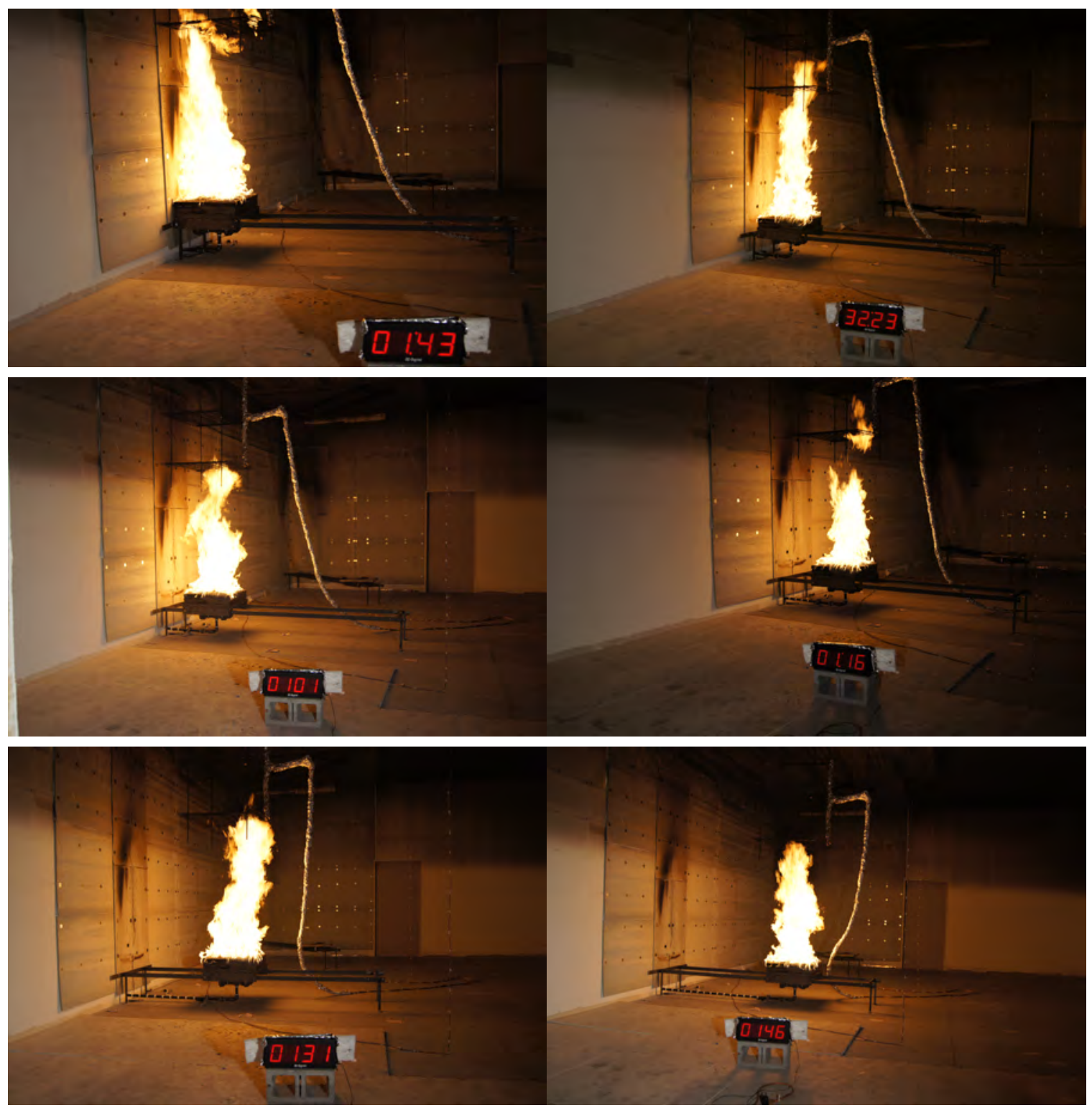

Fig. 10. Photographs of $400 \mathrm{~kW}$ wall fire at distances from the wall of $0 \mathrm{~cm}, 10 \mathrm{~cm}(4 \mathrm{in}), 30 \mathrm{~cm}$ (12 in), $50 \mathrm{~cm}$ (20 in), $100 \mathrm{~cm}$ (40 in), and $160 \mathrm{~cm}$ (63 in). 


\subsection{Results}

The two key results for these experiments is the plume temperature, as measured by the 3-D thermocouple (TC) array above the burner; and the hot gas layer (HGL) temperature and height, as derived from the centerline vertical TC arrays.

\subsubsection{Plume Temperatures}

In each experiment, as the burner and TC array moved from position to position, the location of the plume centerline with respect to the TCs would also change. In order to determine the peak or centerline plume temperature at each of the three levels, the individual TC temperatures were first time-averaged ${ }^{7}$ over a minute to eliminate spikes in the data, and then the maximum value of the averaged temperatures was chosen for each of the three levels. Figures 11 and 12 display the plume temperatures at heights of $1.6 \mathrm{~m}(5 \mathrm{ft}$,

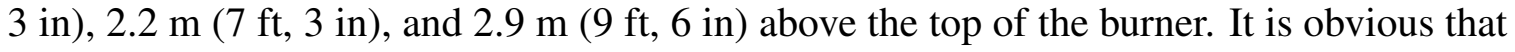
the corner has a noticeable effect on the plume temperature as the burner is moved away, but there is no such trend for the wall fire. The warming of the plume temperatures in the second hour of the wall fire experiments can be explained by the gradual heating of the compartment.

The horizonal, dashed lines on the plots covering the first $30 \mathrm{~min}$ and final $15 \mathrm{~min}$ of each experiment indicate temperatures calculated using Heskestad's plume correlation, Eq. 1. For the initial $30 \mathrm{~min}$, when the fire is right up against the wall or corner, respectively, Eq. 1 is evaluated with twice or four times the heat release rate and base area (i.e. $D$ is multiplied by $\sqrt{2}$ and 2, respectively). For the final $15 \mathrm{~min}$, Eq. 1 is evaluated using the actual heat release rate and fire base area. The base diameter, $D$, of the $0.6 \mathrm{~m}$ by $0.6 \mathrm{~m}$ ( $2 \mathrm{ft}$ by $2 \mathrm{ft}$ ) square burner is taken as $D=\sqrt{4 A / \pi} \approx 0.68 \mathrm{~m}$ ( $2 \mathrm{ft}, 3 \mathrm{in}$ ). In all cases, the radiative fraction of the fire is assumed to be 0.25 ; that is, the convective heat release rate, $\dot{Q}_{c}$, is assumed to be $75 \%$ of the total heat release rate [9]. No attempt has been made to account for the effect of the hot gas layer or ceiling when evaluating Heskestad's correlation, which can explain some of the differences with the actual measurements.

\footnotetext{
${ }^{7}$ The time-averaging was achieved by averaging values over a time increment of $\pm 30 \mathrm{~s}$.
} 

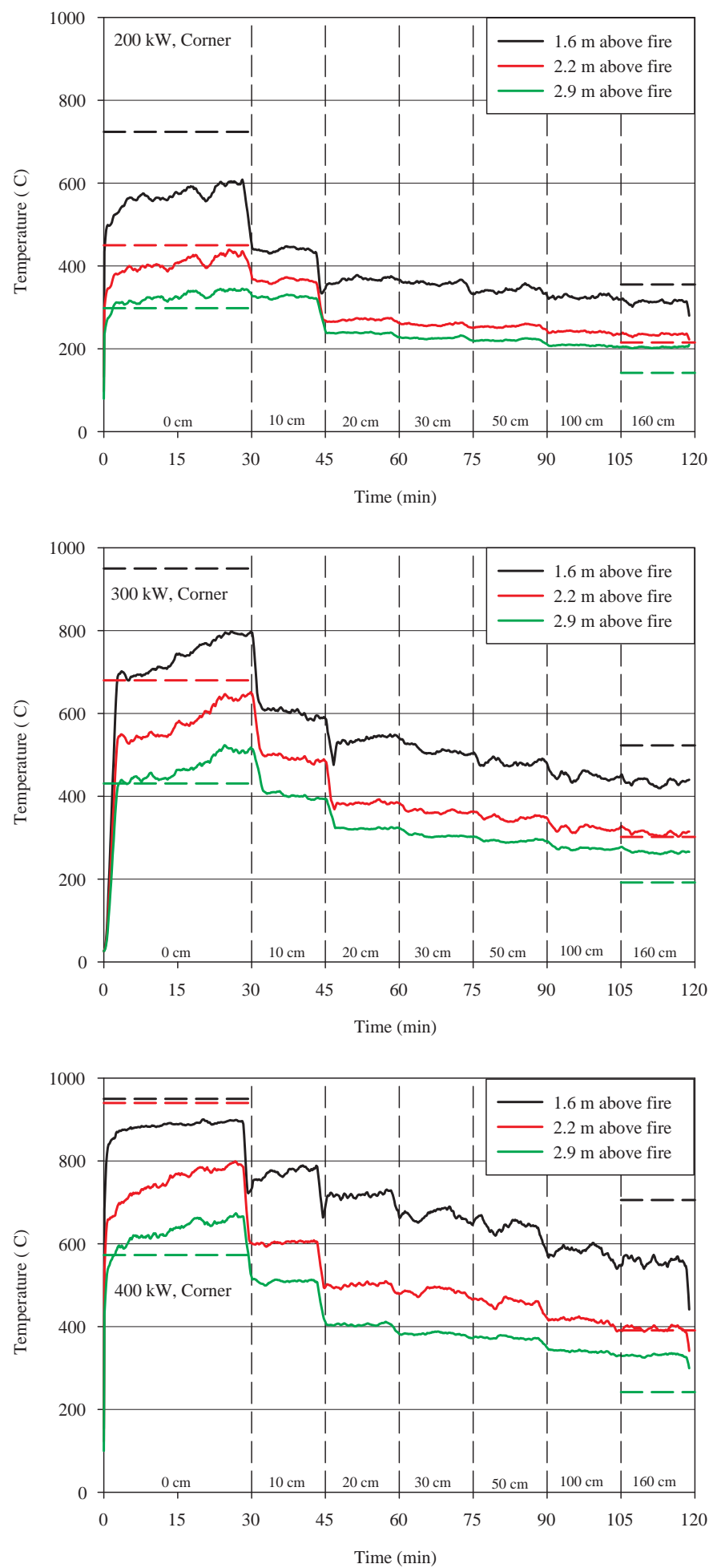

Fig. 11. Plume temperature at three heights above $200 \mathrm{~kW}, 300 \mathrm{~kW}$, and $400 \mathrm{~kW}$ corner fires, for various distances from the corner. The horizontal dashed lines indicate the results of the Heskestad correlation, Eq. 1. 

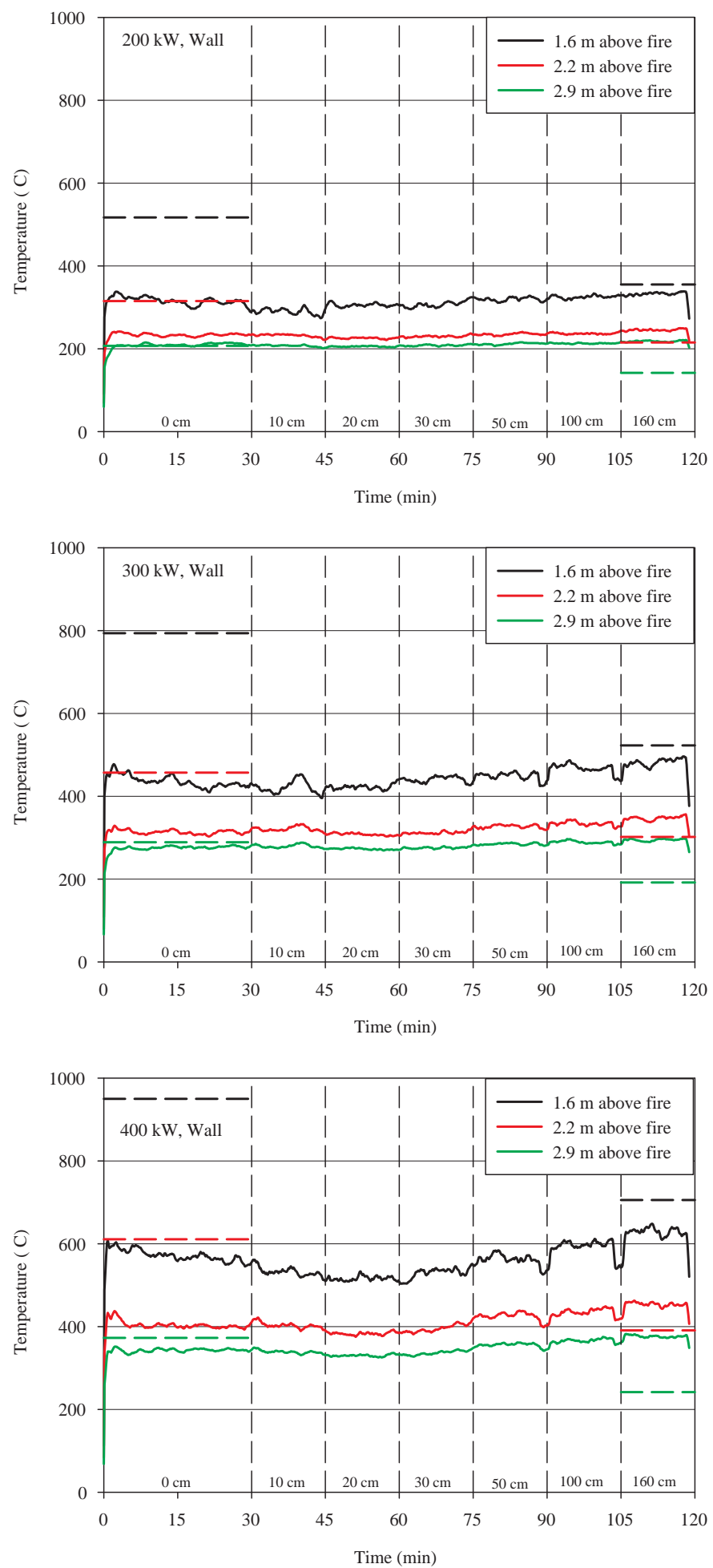

Fig. 12. Plume temperature at three heights above $200 \mathrm{~kW}, 300 \mathrm{~kW}$, and $400 \mathrm{~kW}$ wall fires, for various distances from the wall. The horizontal dashed lines indicate the results of the Heskestad correlation, Eq. 1. 


\subsubsection{Hot Gas Layer Temperature and Height}

The two vertical thermocouple arrays located one-third and two-thirds of the distance along the long compartment centerline were used to compute hot gas layer (HGL) temperatures and heights for each experiment.

Figures 13 and 14 display the HGL temperature and height for the six experiments. There does not appear to be a significant change in either quantity as the burner is moved away from the corner or wall. The gradual increase in HGL temperature and decrease in HGL height are typical of any steady compartment fire. 

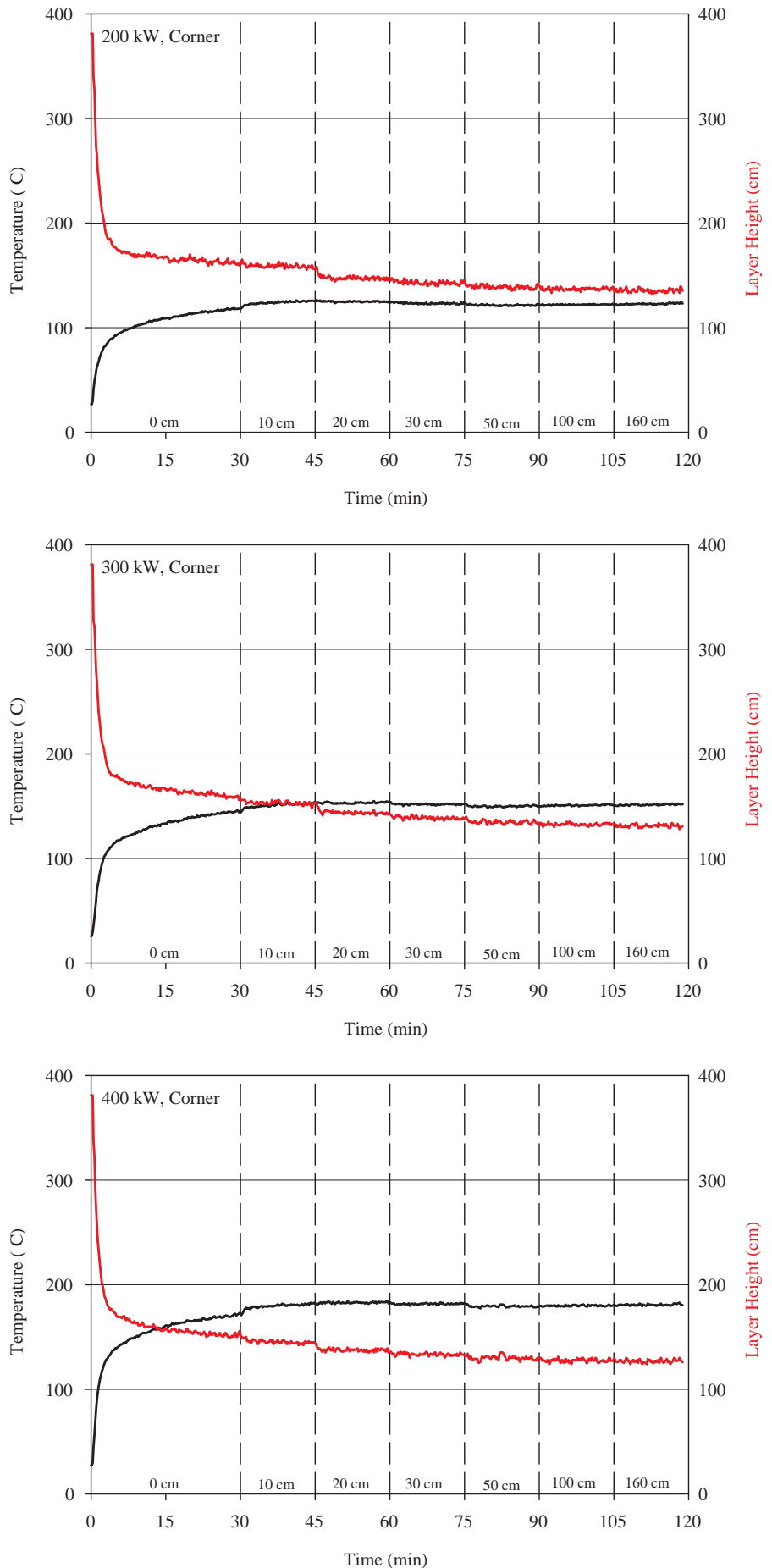

Fig. 13. HGL temperature (black) and height (red) for $200 \mathrm{~kW}, 300 \mathrm{~kW}$, and $400 \mathrm{~kW}$ corner fires, for various distances from the corner. 

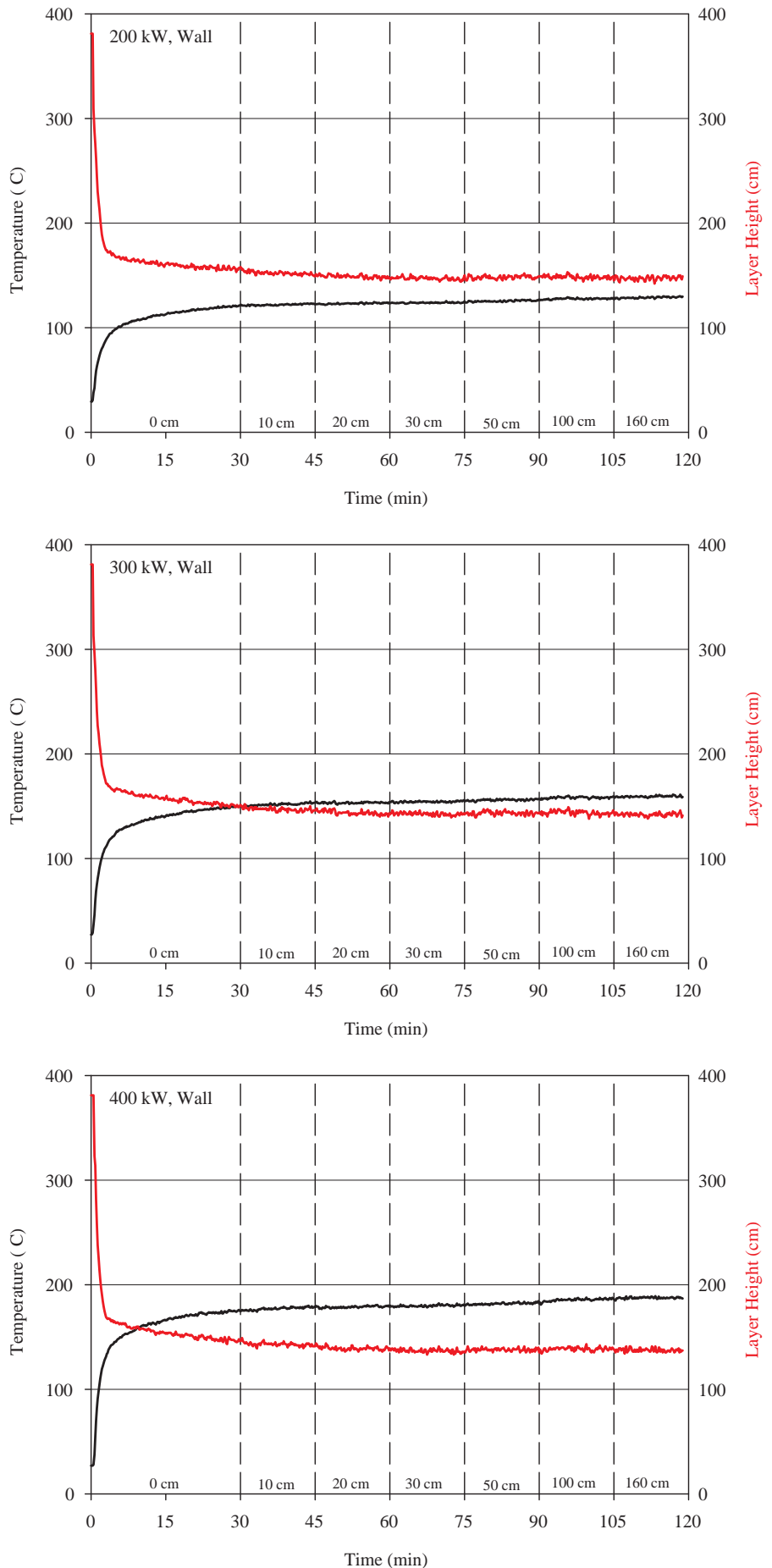

Fig. 14. HGL temperature (black) and height (red) for $200 \mathrm{~kW}, 300 \mathrm{~kW}$, and $400 \mathrm{~kW}$ wall fires, for various distances from the wall. 


\section{Cabinet Experiments}

In this second series of experiments, conducted in September, 2017, natural gas burners were placed within two different mock steel cabinets to assess their effect on the compartment temperatures.

\subsection{Description of Cabinets}

The steel cabinets used in the experiments were designed as simple models of electrical enclosures found in industrial settings, in particular nuclear power plants. Each cabinet was constructed of 12 gauge ( $2.8 \mathrm{~mm}$ or 7/64 in) steel plate with openings as shown in

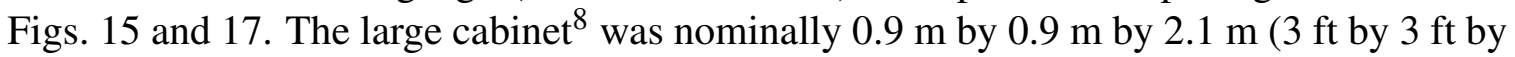
$7 \mathrm{ft}$ ) and the medium size cabinet was $0.6 \mathrm{~m}$ by $0.6 \mathrm{~m}$ by $2.1 \mathrm{~m}(2 \mathrm{ft}$ by $2 \mathrm{ft}$ by $7 \mathrm{ft})$. The openings near the top of each cabinet were sometimes covered with a steel grill, shown in Fig. 19, and sometimes covered by a solid plate. Note that the cabinets were designed and built based on the specified Imperial rather than metric units. The precision of the steel cutting equipment is approximately $0.8 \mathrm{~mm}(1 / 32 \mathrm{in})$.

${ }^{8}$ The description of the cabinets as "large" and "medium-sized" is based on the characterization of electrical enclosures used by the U.S. Nuclear Regulatory Commission in Ref. [3]. 


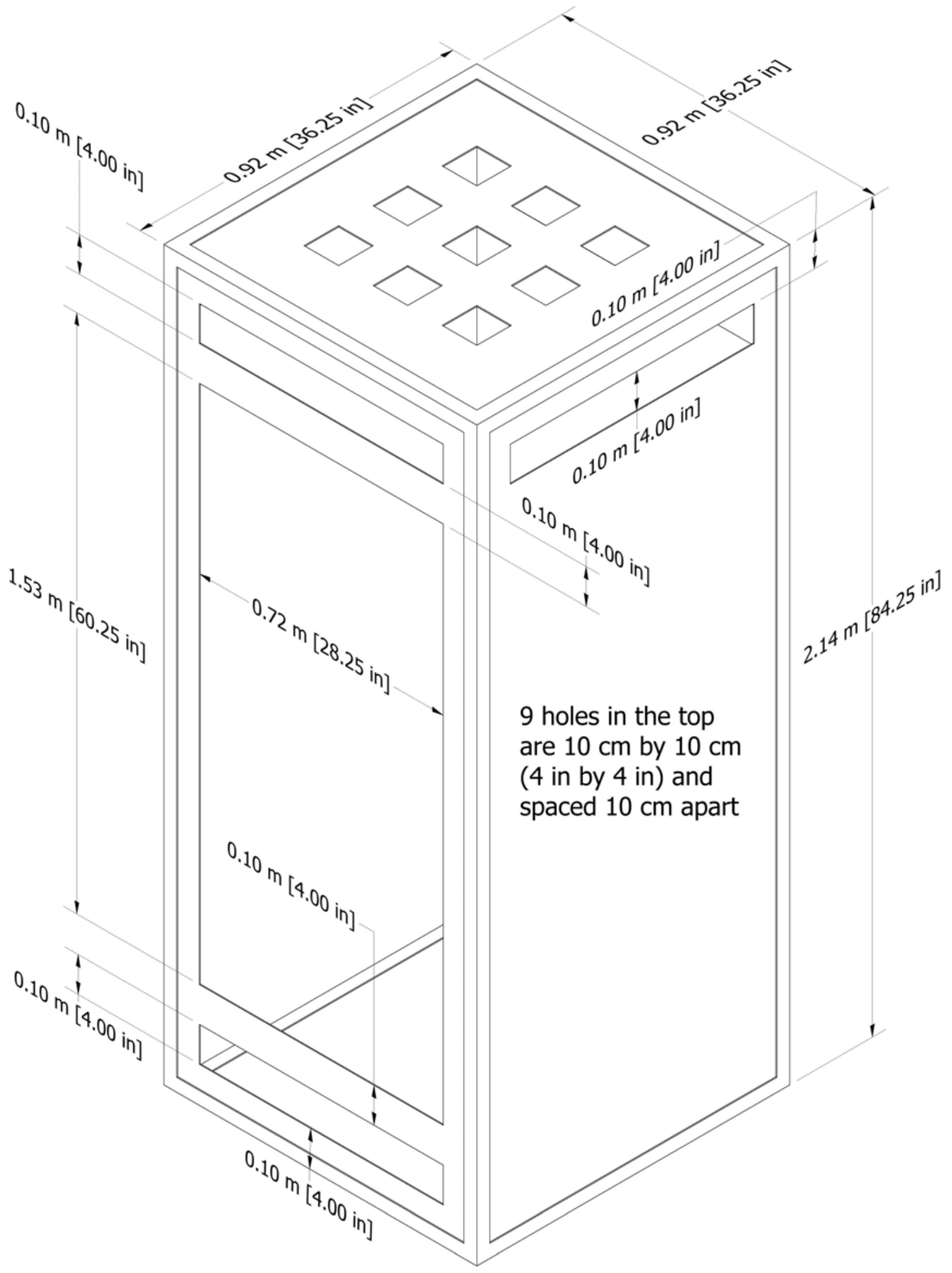

Fig. 15. Large cabinet drawing, NIST/NRC Corner Effects Experiments. 


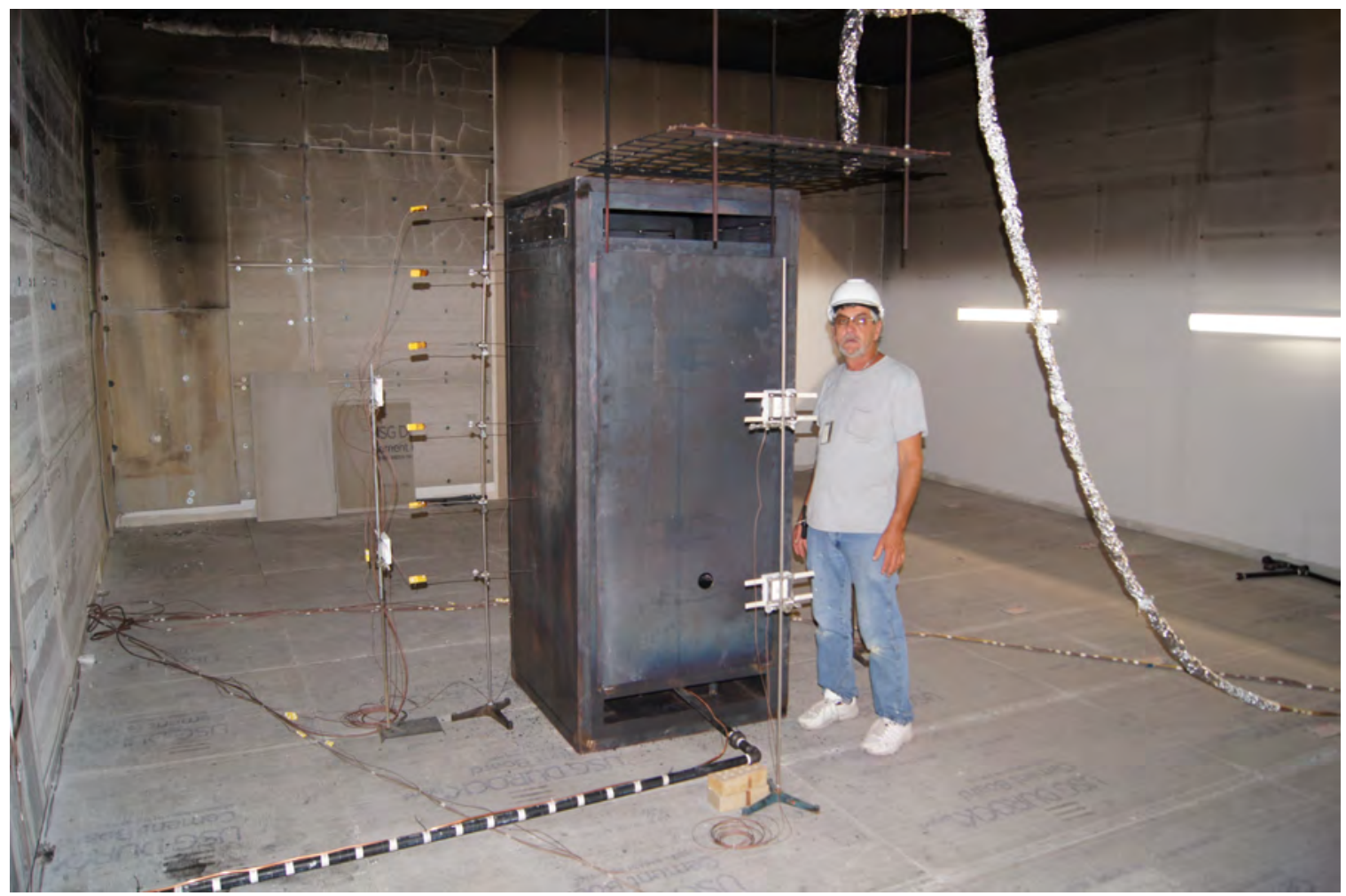

Fig. 16. Photograph of the large cabinet. Note the position of the thermocouple array just above the front face of the cabinet. 


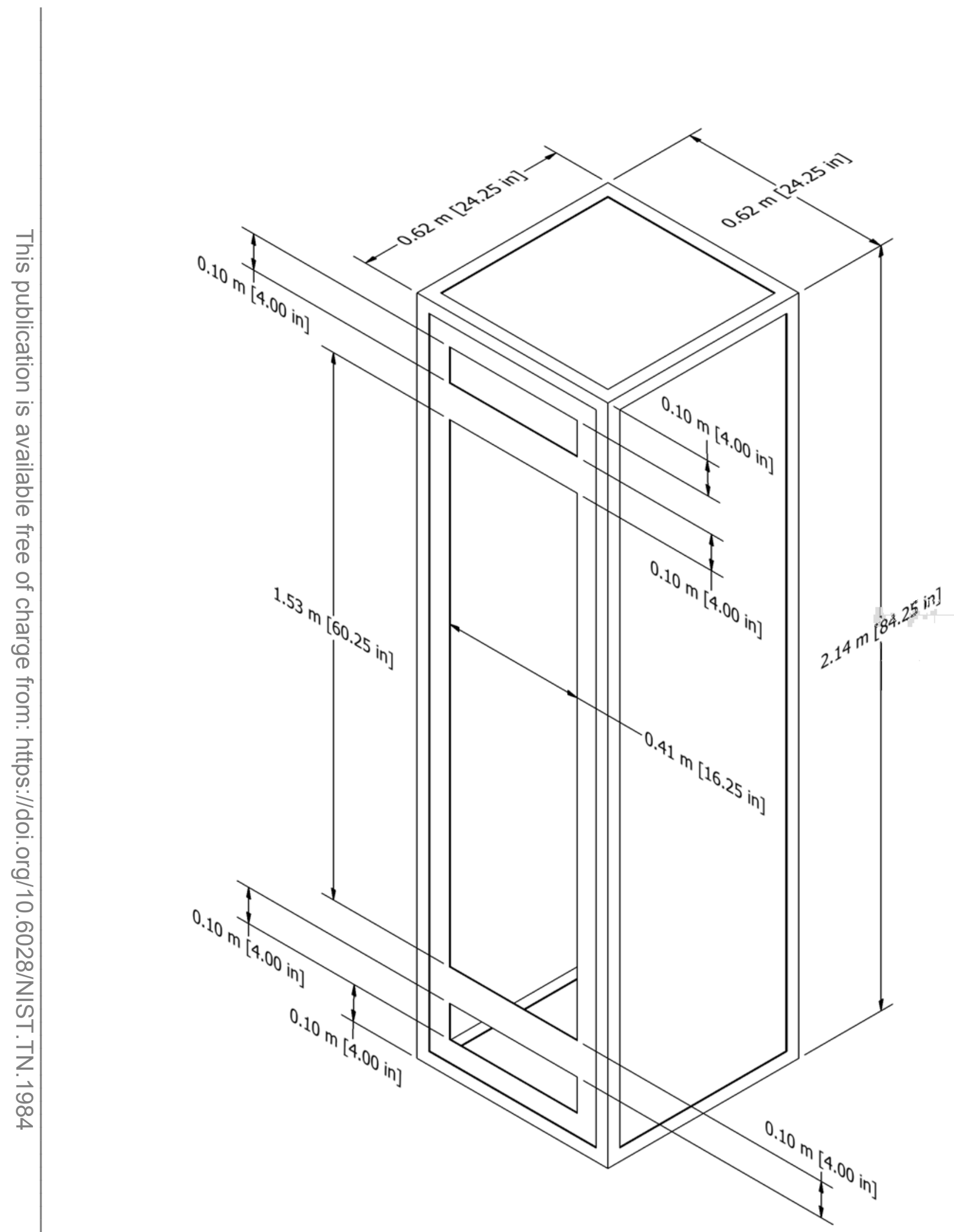

Fig. 17. Medium-sized cabinet drawing, NIST/NRC Corner Effects Experiments. 


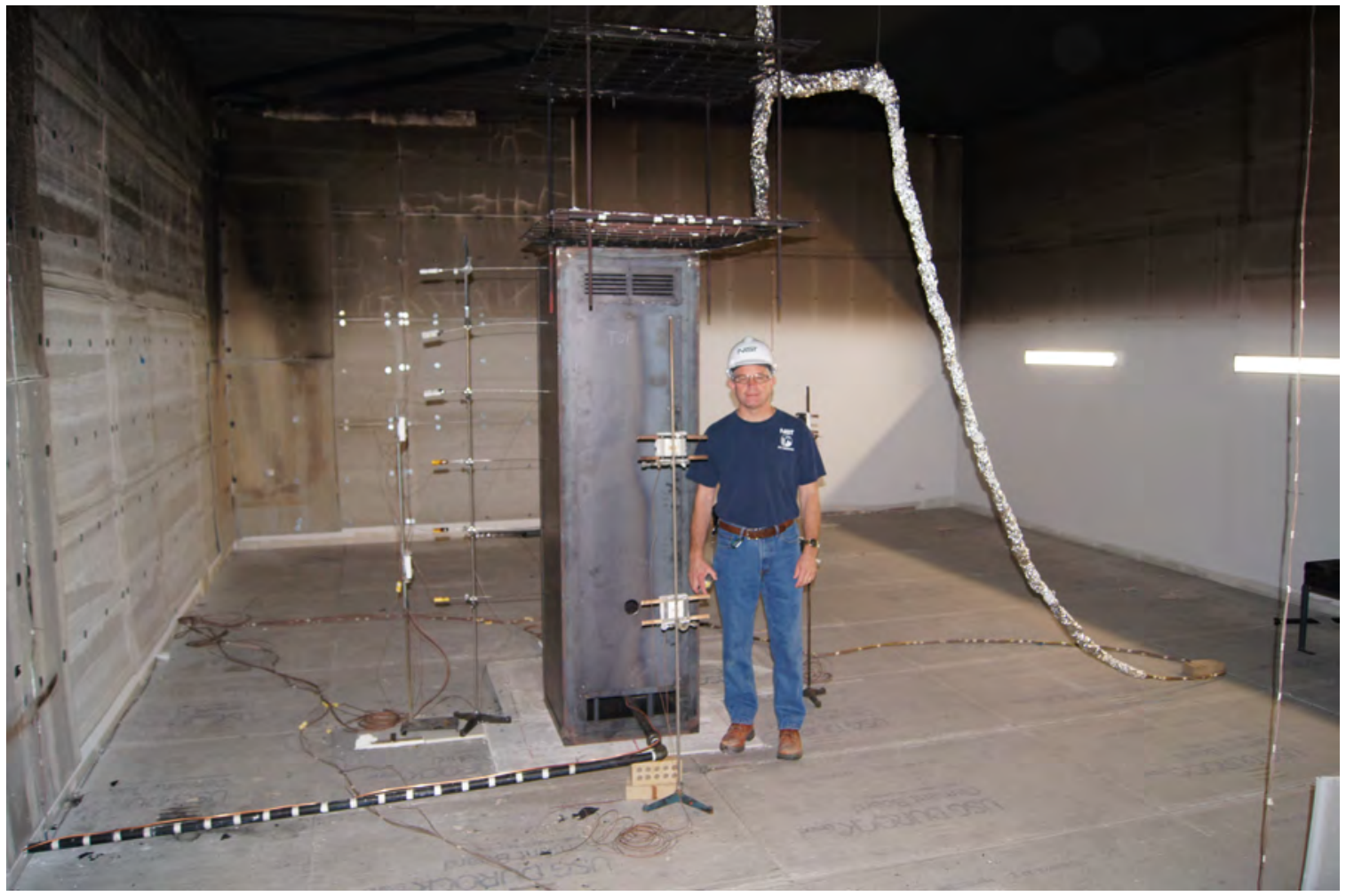

Fig. 18. Photograph of the medium-sized cabinet. Note the position of the thermocouple array just above the front face of the cabinet. 

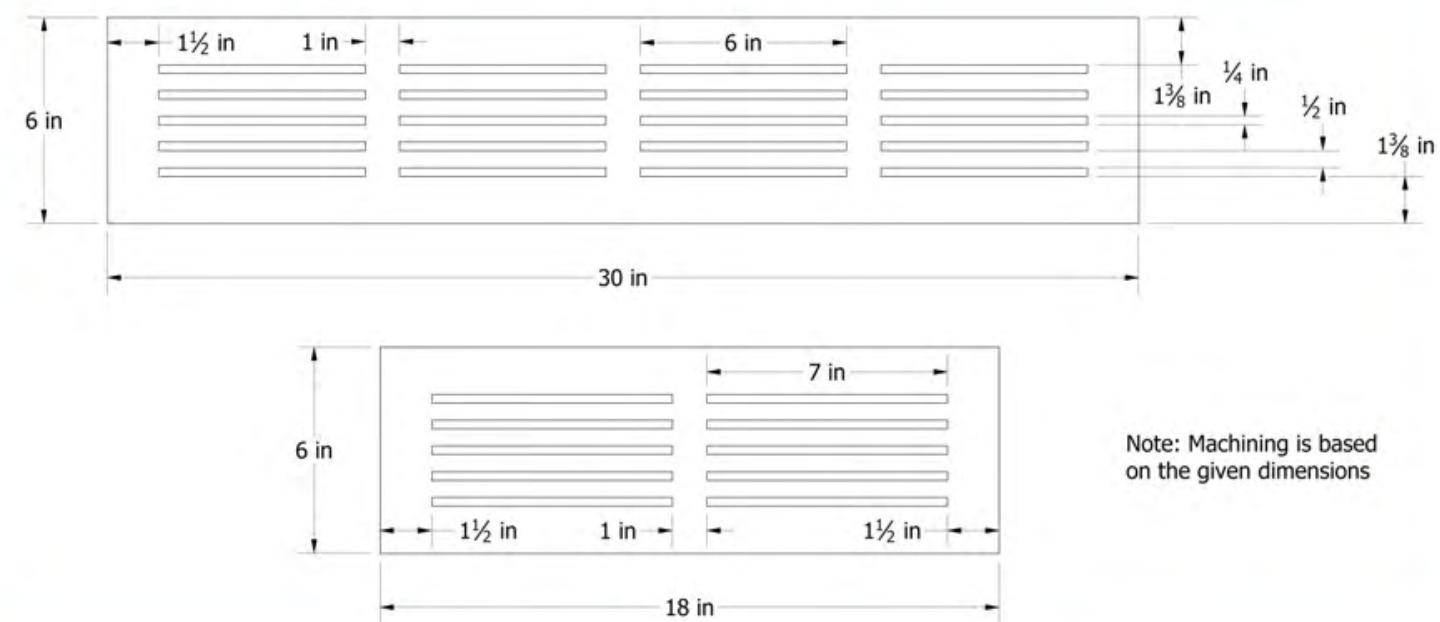

Note: Machining is based on the given dimensions

Fig. 19. Cabinet grill, NIST/NRC Corner Effects Experiments.

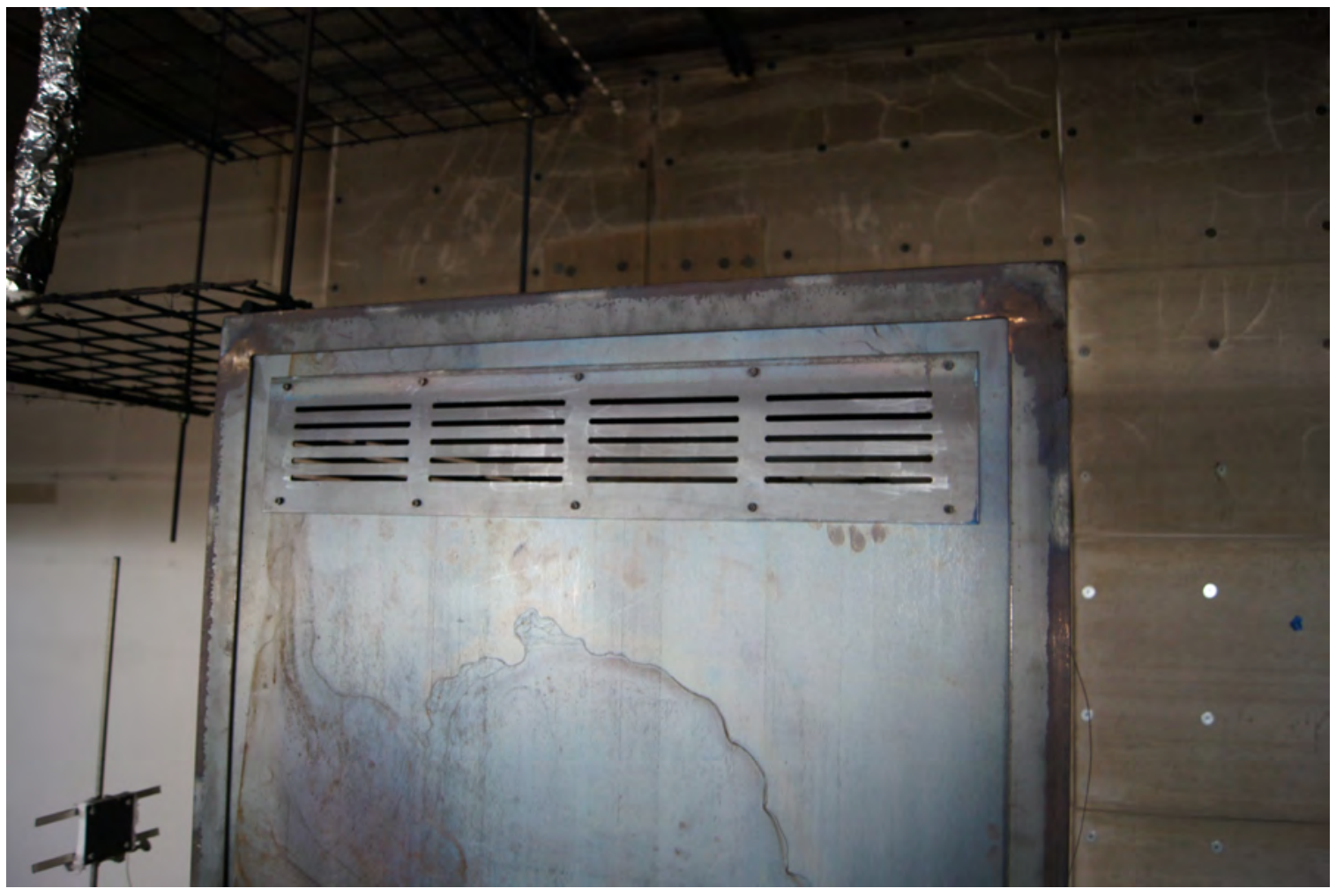

Fig. 20. Photograph of the grill over the large cabinet upper vent. Note that the grill covers approximately three-fourths of the opening area. 


\subsection{Procedure}

Figure 21 presents a schematic diagram of the cabinet experiments. Table 1 lists the test parameters. For the first set of experiments (Tests 1-6), the large cabinet was positioned with its front opening facing eastward towards the opening of the test compartment. Its left side was $1.8 \mathrm{~m}(6 \mathrm{ft})$ from the south wall and its front side was $5.8 \mathrm{~m}(19 \mathrm{ft})$ from the east wall. Two $0.3 \mathrm{~m}$ by $0.3 \mathrm{~m}$ ( $1 \mathrm{ft}$ by $1 \mathrm{ft}$ ) natural gas burners were placed side by side in the cabinet from the perspective of the cabinet front opening. The top of the burner was $50 \mathrm{~cm}$ (20 in) above the floor of the cabinet. For Tests 1-4, the front door of the cabinet was closed, and the heat release rate was initially set to $50 \mathrm{~kW}$ for $30 \mathrm{~min}$, then it was increased to $100 \mathrm{~kW}$ for $15 \mathrm{~min}, 200 \mathrm{~kW}$ for $15 \mathrm{~min}$, and $400 \mathrm{~kW}$ for $15 \mathrm{~min}$. Figure 22 shows the large cabinet with a $400 \mathrm{~kW}$ fire near the end of Test 2.

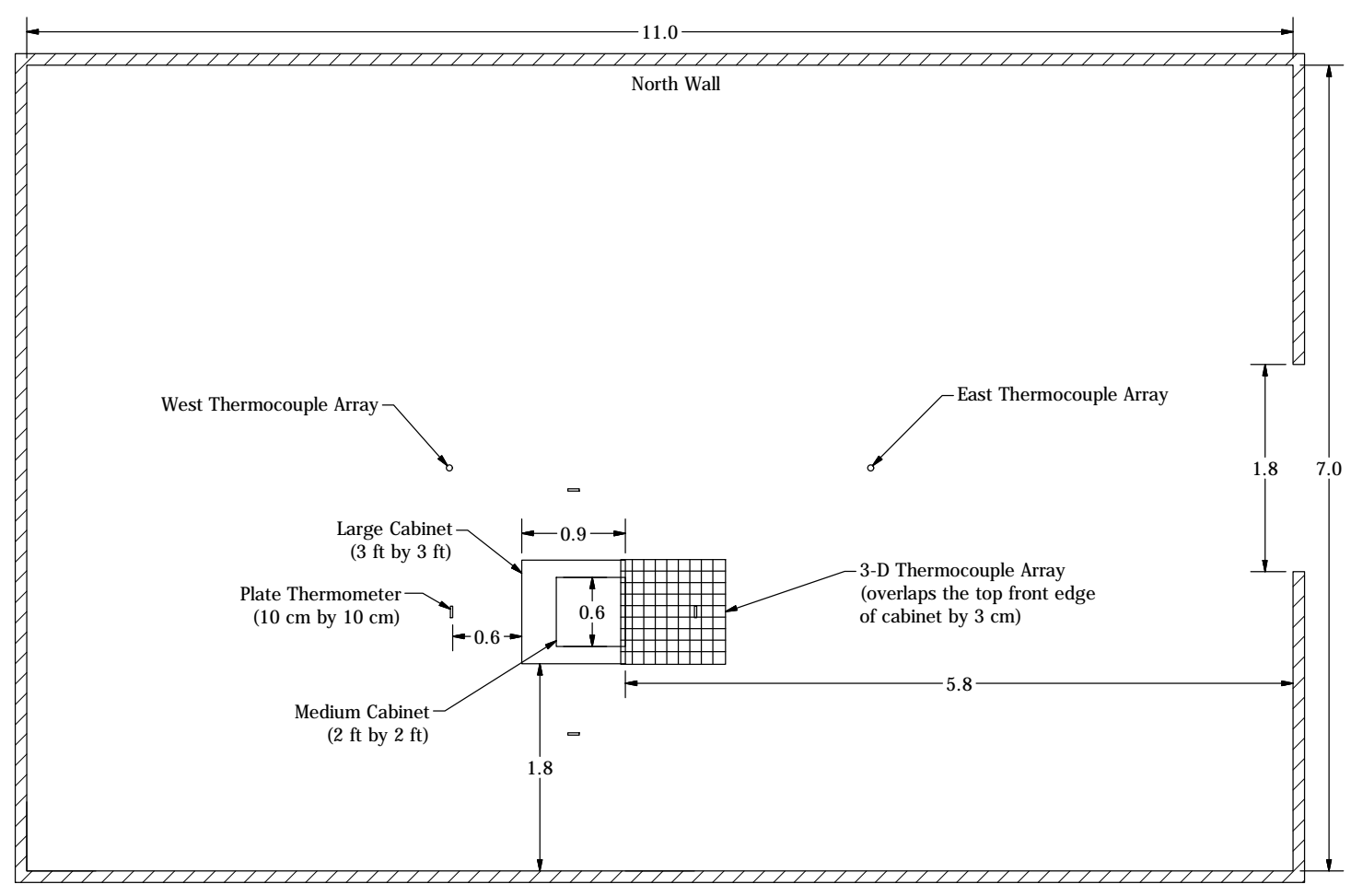

Fig. 21. Plan view of the compartment showing locations of the cabinets. All dimensions are in meters.

For Tests 5-6, the front door was opened, and the heat release rate was set to $200 \mathrm{~kW}$, $400 \mathrm{~kW}$, and $700 \mathrm{~kW}$ for $15 \mathrm{~min}$ each, and then $1000 \mathrm{~kW}$ for $5 \mathrm{~min}$, a total of $50 \mathrm{~min}$. 
Table 1. Summary of Cabinet Experiments.

\begin{tabular}{|c|c|c|c|l|l|}
\hline Test & Cabinet & Front Door & Top Vents & Upper Side Vents & HRR (kW) \\
\hline \hline 1 & Large & Closed & Closed & Grill on four sides & $50,100,200,400$ \\
\hline 2 & Large & Closed & All open & Grill on four sides & $50,100,200,400$ \\
\hline 3 & Large & Closed & Closed & Front open, all others closed & $50,100,200,400$ \\
\hline 4 & Large & Closed & Closed & Front, back open, others closed & $50,100,200,400$ \\
\hline 5 & Large & Open & Closed & Front, back open, others closed & $200,400,700,1000$ \\
\hline 6 & Large & Open & All open & All open & $200,400,700,1000$ \\
\hline 7 & Medium & Closed & N/A & Grill & $25,50,100,200$ \\
\hline 8 & Medium & Closed & N/A & Open & $25,50,100,200$ \\
\hline 9 & Medium & Open & N/A & Open & $40,80,200,325$ \\
\hline 10 & Medium & Open & N/A & Closed & $40,80,200,325$ \\
\hline 11 & None & N/A & N/A & N/A & $200,400,700,1000$ \\
\hline 12 & None & N/A & N/A & N/A & $50,100,200,400$ \\
\hline
\end{tabular}

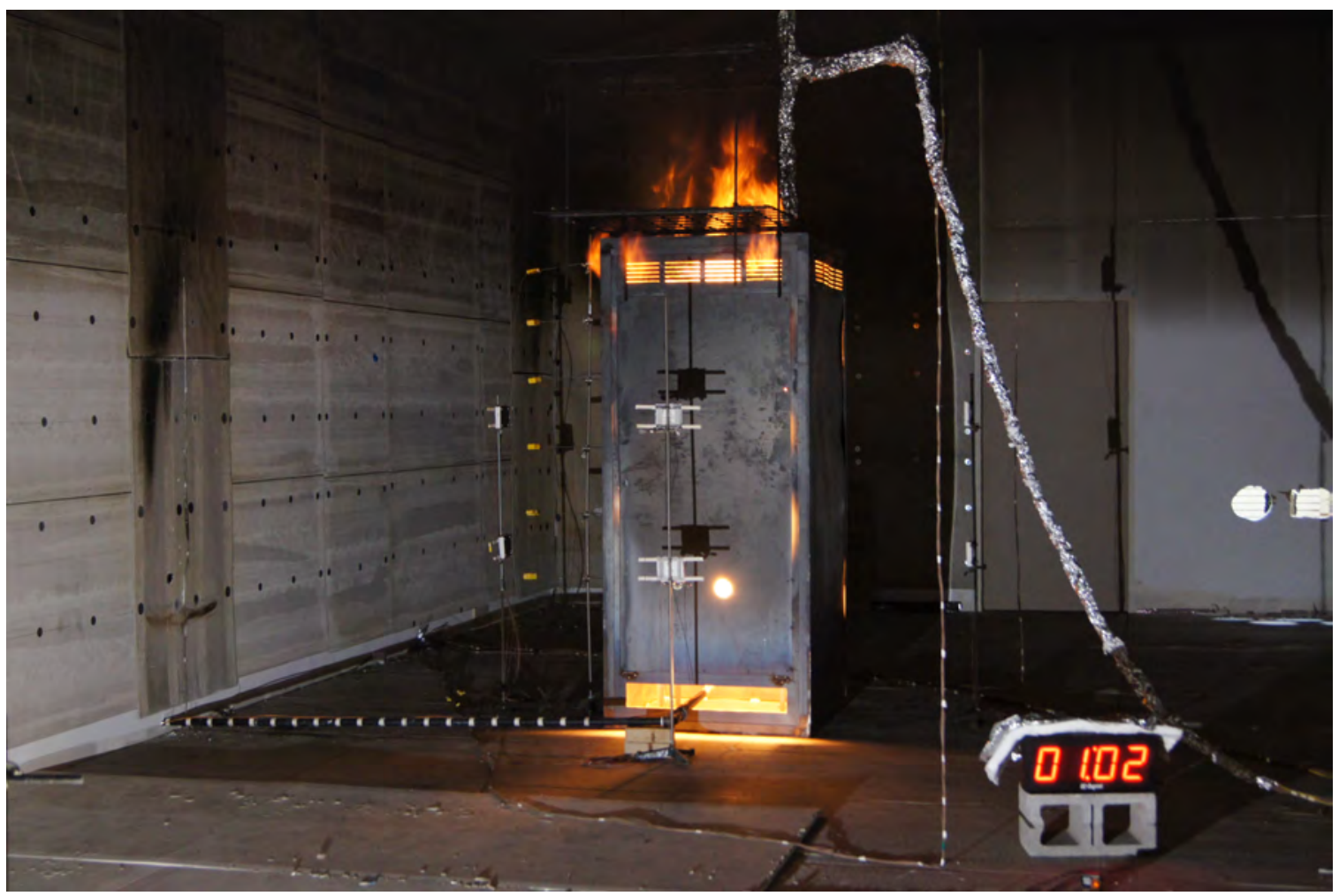

Fig. 22. Photograph of large cabinet with $400 \mathrm{~kW}$ fire (Test 2). 


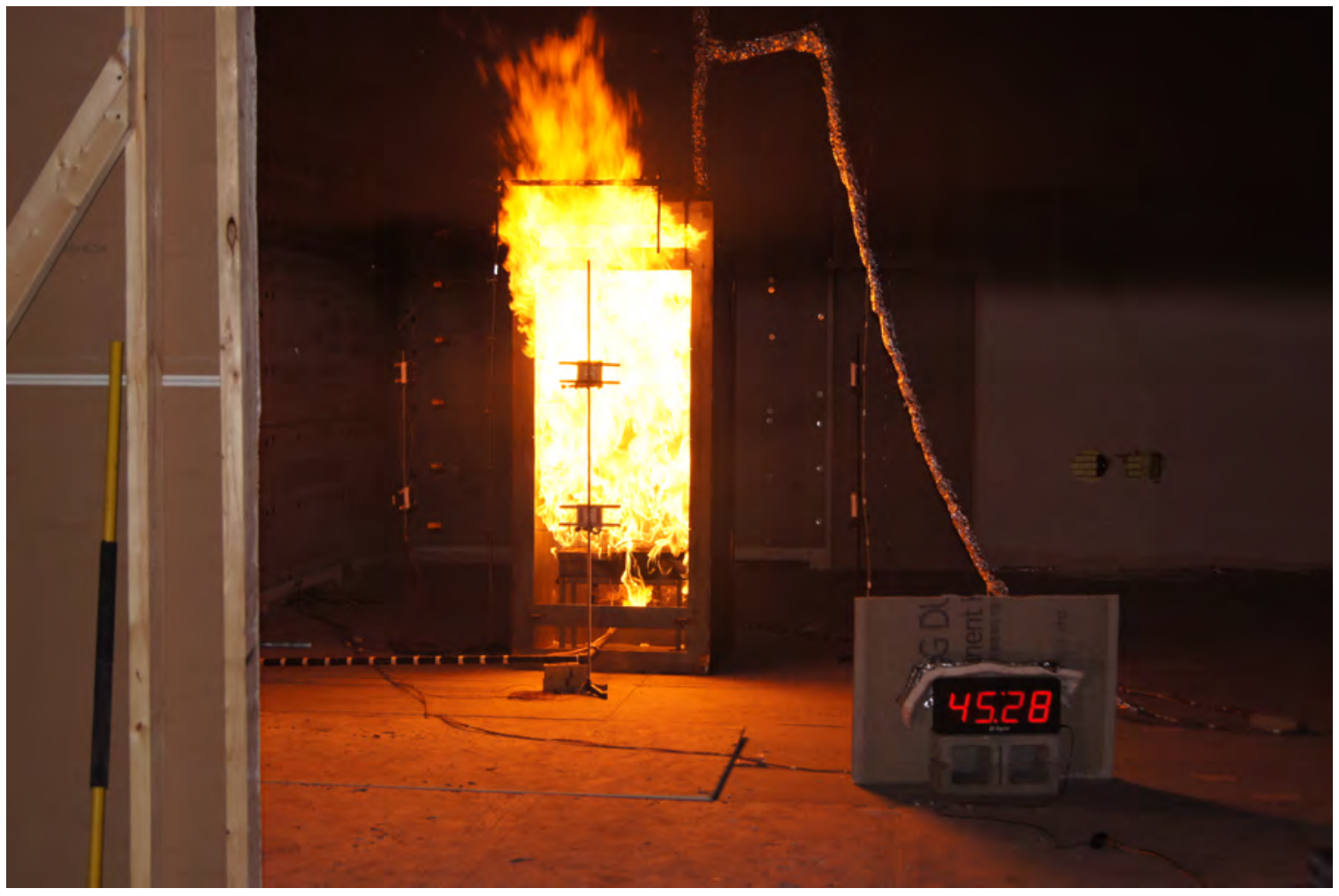

Fig. 23. Photograph of large cabinet with $1000 \mathrm{~kW}$ fire (Test 5). 
In the second set of experiments (Tests 7-10), the medium-sized cabinet was positioned so that its front was the same distance from the east wall as the large cabinet, and its left side was $2.0 \mathrm{~m}(6.5 \mathrm{ft})$ from the south wall. A single $30 \mathrm{~cm}$ by $30 \mathrm{~cm}(1 \mathrm{ft}$ by $1 \mathrm{ft})$ gas burner was centered within. For the closed door tests (Tests 7-8), the heat release rate was $25 \mathrm{~kW}, 50 \mathrm{~kW}, 100 \mathrm{~kW}$, and $200 \mathrm{~kW}$, each for $15 \mathrm{~min}$. Figure 24 shows the cabinet with a $200 \mathrm{~kW}$ fire that has become under-ventilated and generates a noticeable layer of smoke in the compartment. Test 7 was the only experiment that exhibited a noticeably underventilated fire. The front door was closed, and the upper front vent was covered by the grill. In Test 8, the grill was removed and the vent was fully opened, in which case the fire did not become noticeably under-ventilated.

For the open door tests in the medium-sized cabinet (Tests 9-10), the heat release rate was $40 \mathrm{~kW}, 80 \mathrm{~kW}, 200 \mathrm{~kW}$, and $325 \mathrm{~kW}$, each for 15 min. Figure 25 shows the cabinet with a $325 \mathrm{~kW}$ fire. 


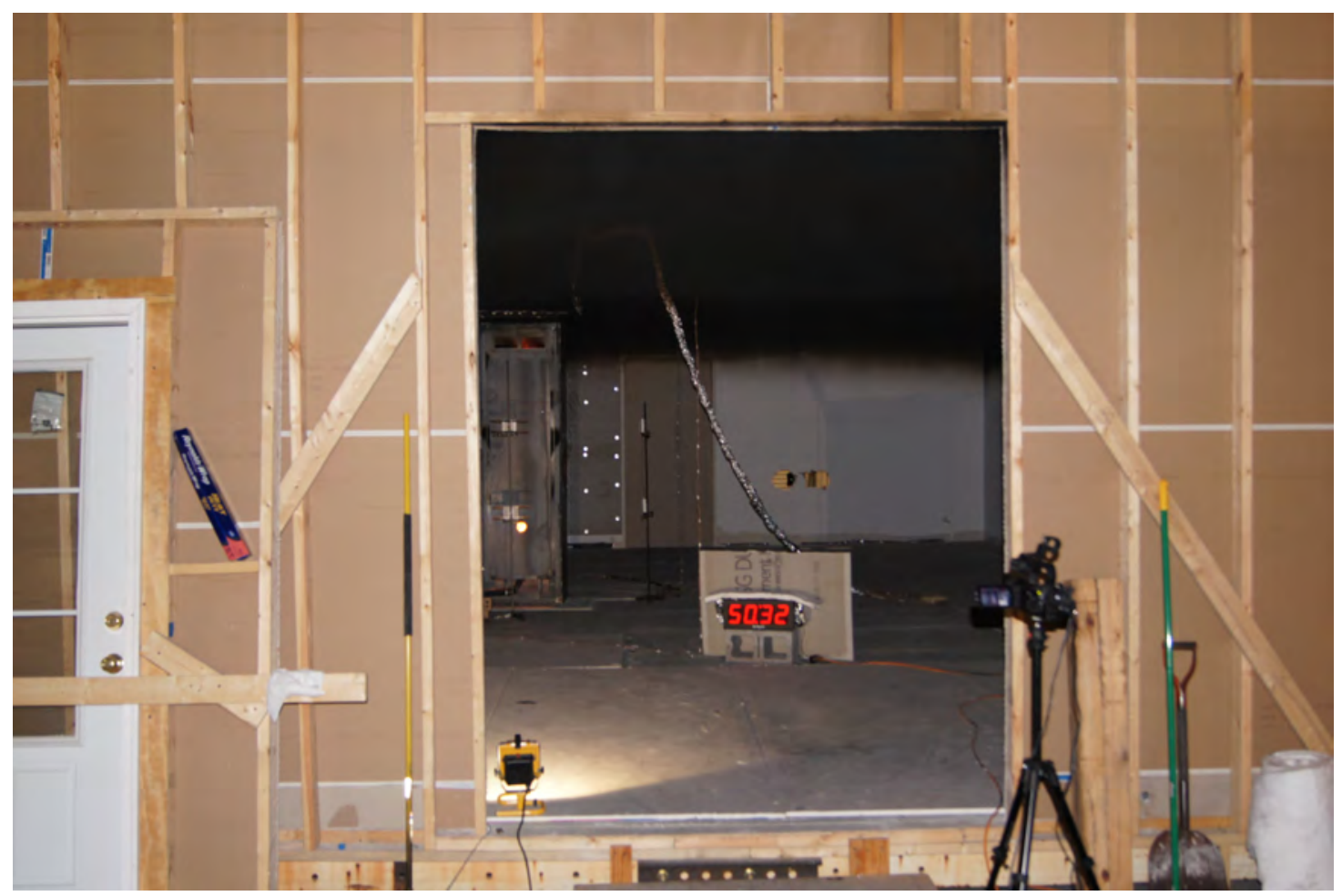

Fig. 24. Photograph of medium cabinet with $200 \mathrm{~kW}$ fire (Test 7).

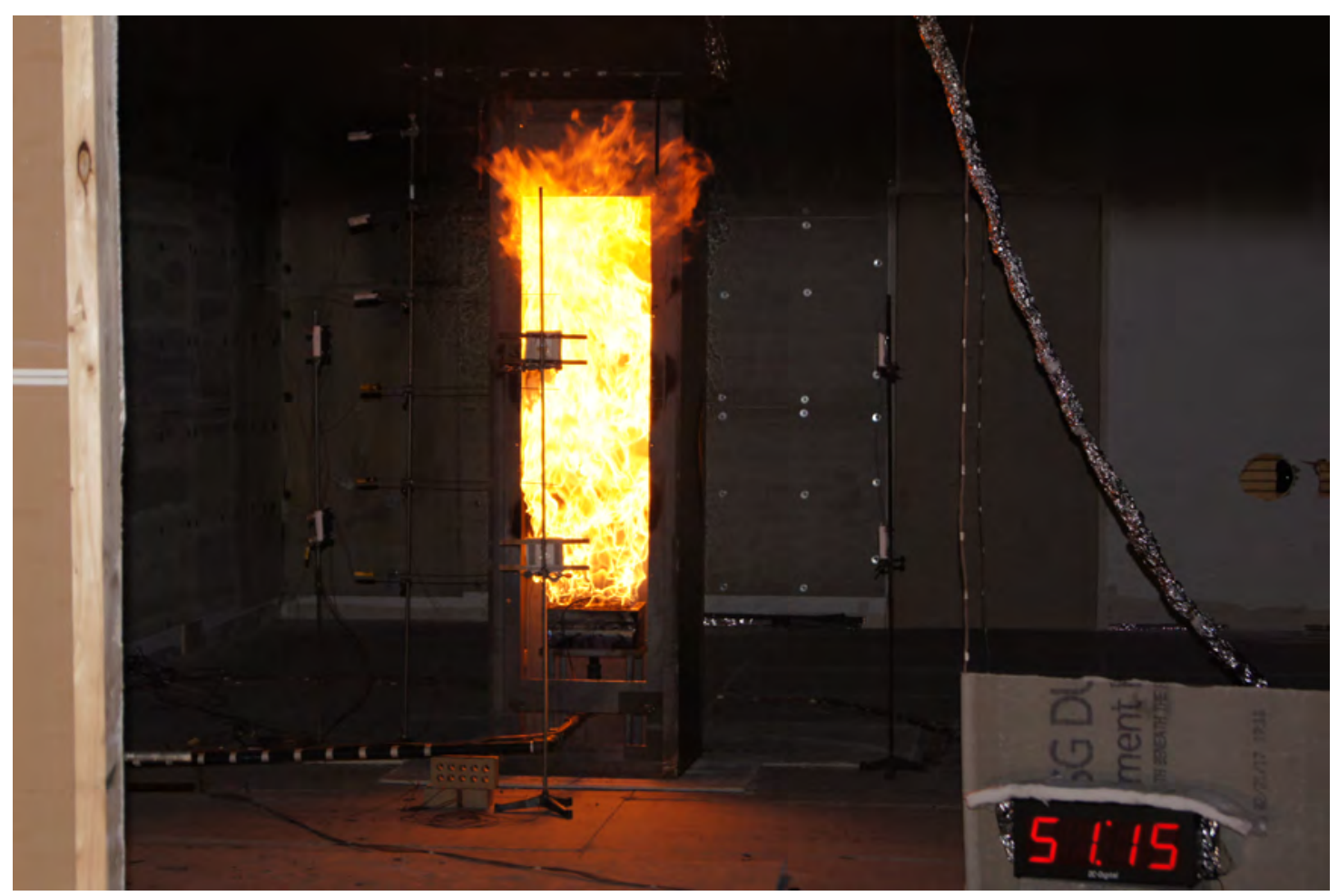

Fig. 25. Photograph of medium cabinet with $325 \mathrm{~kW}$ fire (Test 10). 
In the third set of experiments (Tests 11-12), the cabinet was removed, and two $30 \mathrm{~cm}$

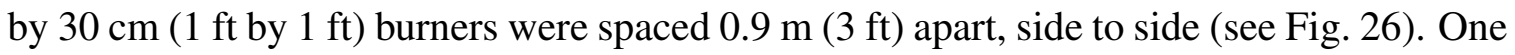
of the burners was centered under the array of thermocouples. Both burners were $2.1 \mathrm{~m}$ $(7 \mathrm{ft})$ from the south wall. These experiments used the same heat release rate sequence as the open and closed door large cabinet experiments.

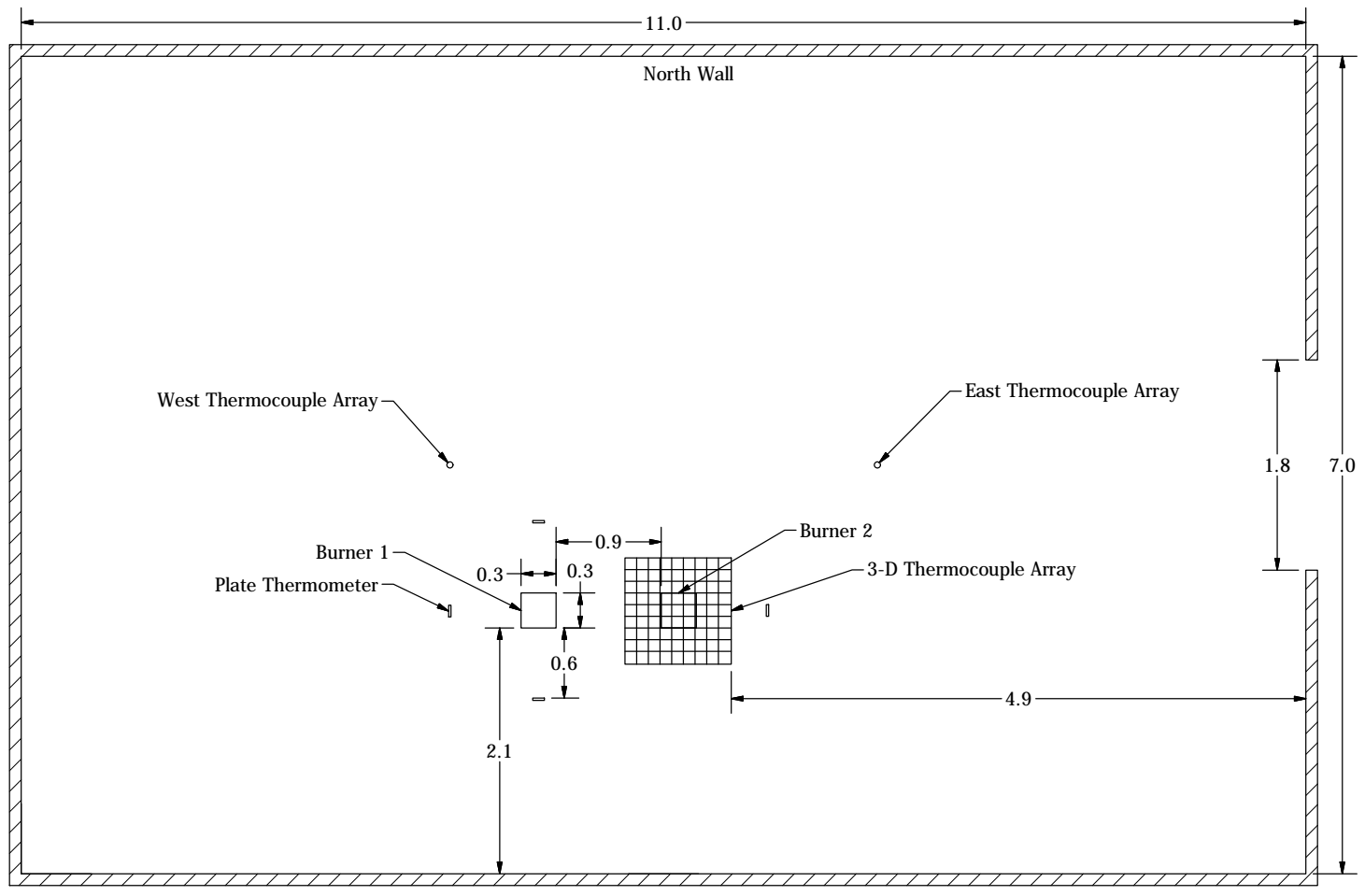

Fig. 26. Plan view of the compartment showing locations of the burners used in Tests 11 and 12 . All dimensions are in meters. 


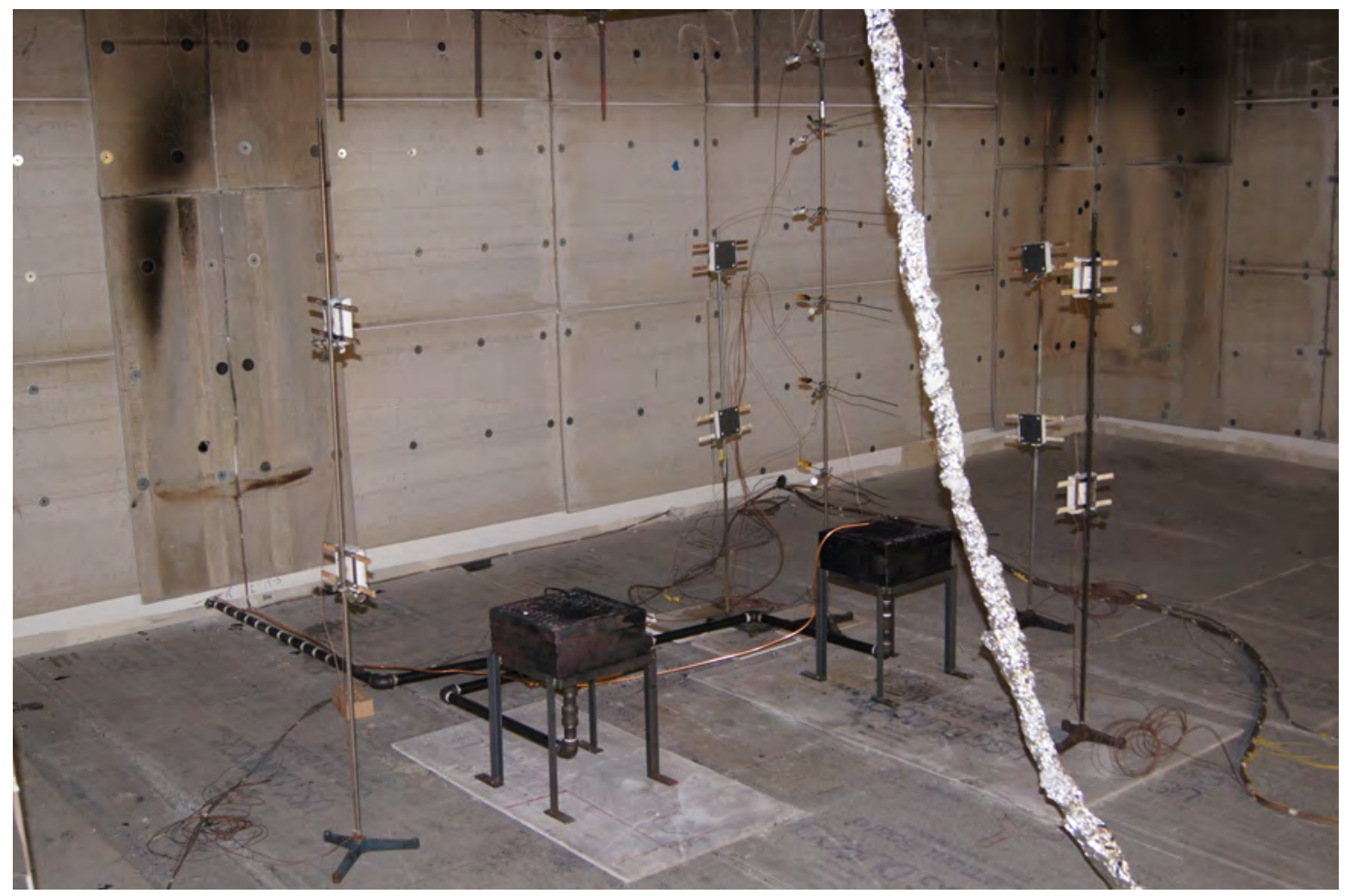

Fig. 27. Configuration of Tests 11 and 12, where the cabinet has been removed.

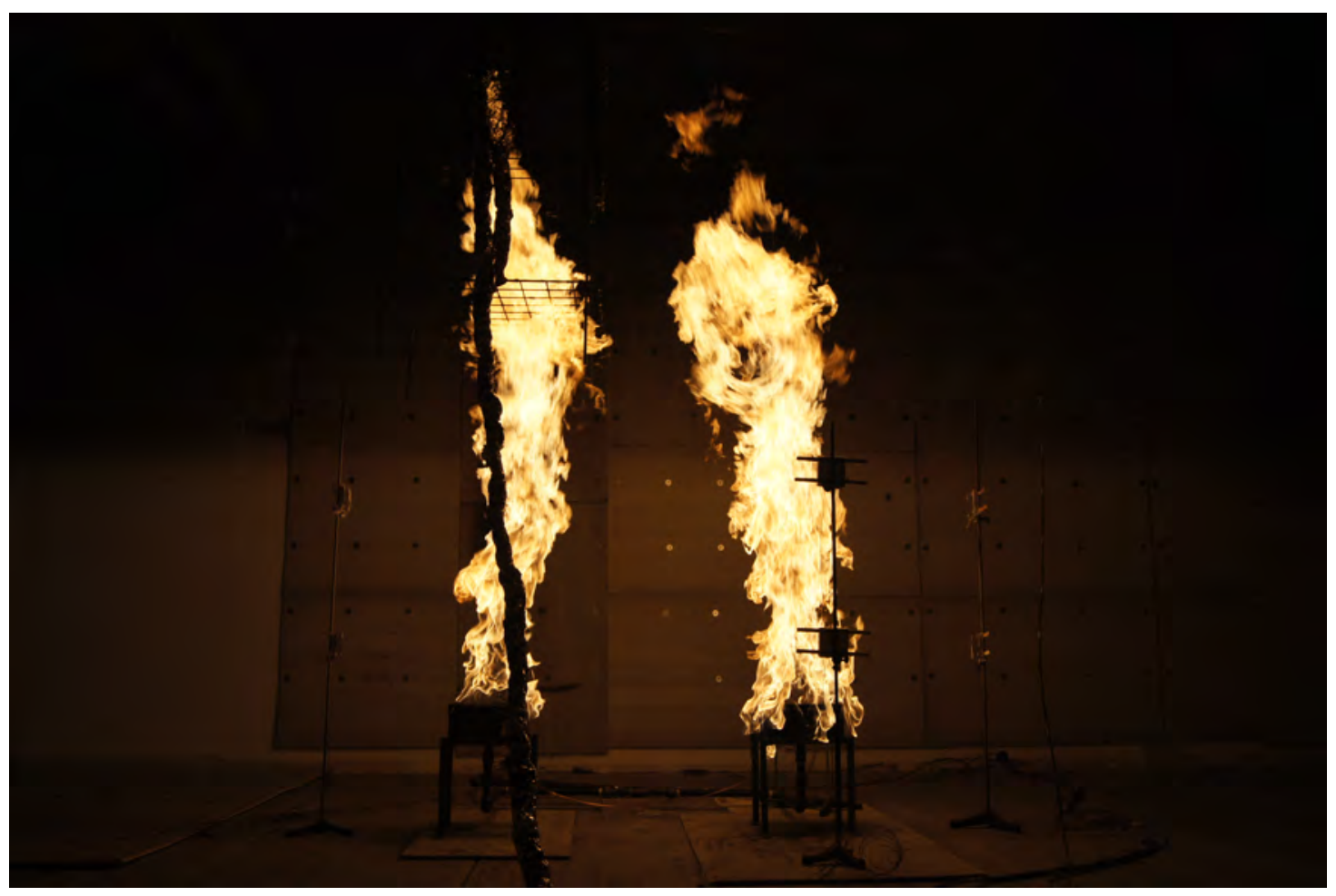

Fig. 28. Photograph of Test 11, $1000 \mathrm{~kW}$. 


\subsection{Results}

The measurements for the cabinet experiments include the inner cabinet gas temperature, cabinet steel wall temperature, near-field heat flux, exhaust plume temperature, and HGL temperature and height. This data is primarily for model validation.

\subsubsection{Cabinet Temperatures and Heat Flux}

Figure 29 displays the near-ceiling gas temperature and the back wall steel temperature for the large cabinet in Test 5 . The peak heat release rate was $1000 \mathrm{~kW}$. The temperatures have been time-averaged over $10 \mathrm{~s}$.

Figure 30 displays the heat flux, as calculated from the plate thermometer temperature, at the upper location of the plates facing the back (black) and front (red) of the open door cabinet. The front plate has an unobstructed view of the flames. In general, the heat flux for the closed door cabinet experiments ranged from $10 \mathrm{~kW} / \mathrm{m}^{2}$ to $20 \mathrm{~kW} / \mathrm{m}^{2}$. 


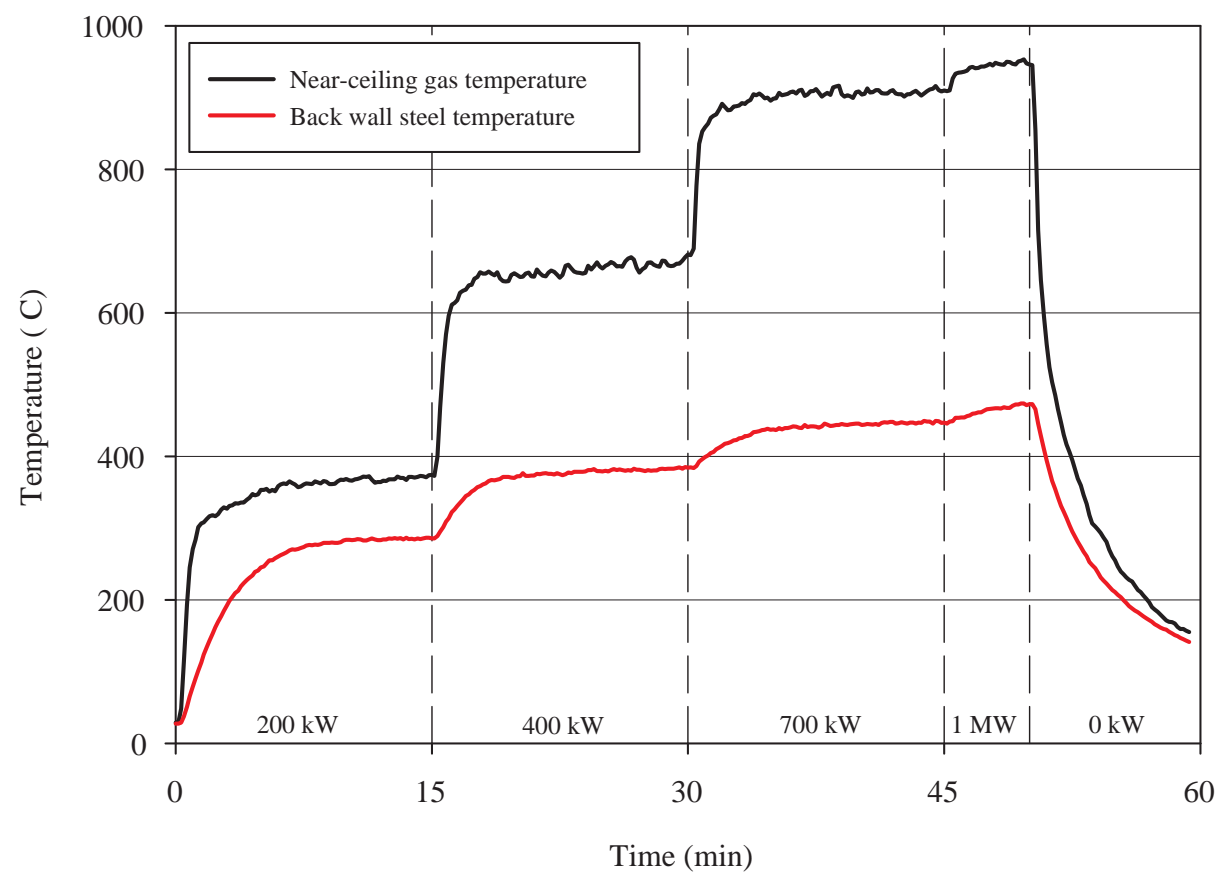

Fig. 29. Gas and steel temperature of the large cabinet, Test 5.

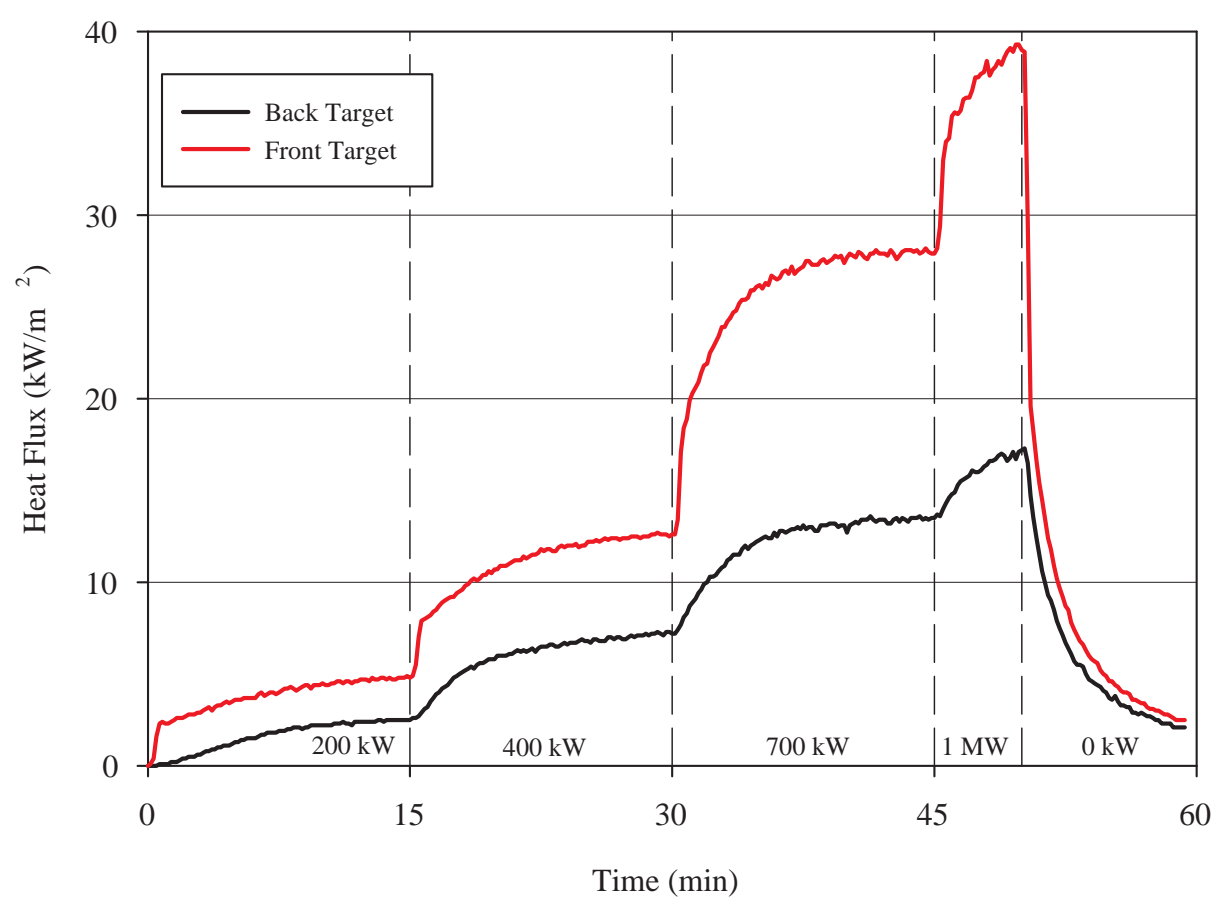

Fig. 30. Heat flux to nearby targets, Test 5 . 


\subsubsection{Ventilation Effects}

The medium-sized cabinet had openings only on its front side. For the closed door experiments, Tests $7-8$, there were only two $10 \mathrm{~cm}$ by $41 \mathrm{~cm}$ (4.00 in by $16.25 \mathrm{in})$ vents, one near the bottom and one near the top. In Test 7, the upper vent was covered by a grill (Fig. 19). As shown in Fig. 31, the limited openings cause the fire to become under-ventilated; that is, the measured heat release rate based on oxygen consumption calorimetry is significantly less than that calculated from the measured flow rate of natural gas to the burner. In essence, the HRR of Test 7 does not rise above $100 \mathrm{~kW}$, and the consequence of this on room temperature is shown in Fig. 32 


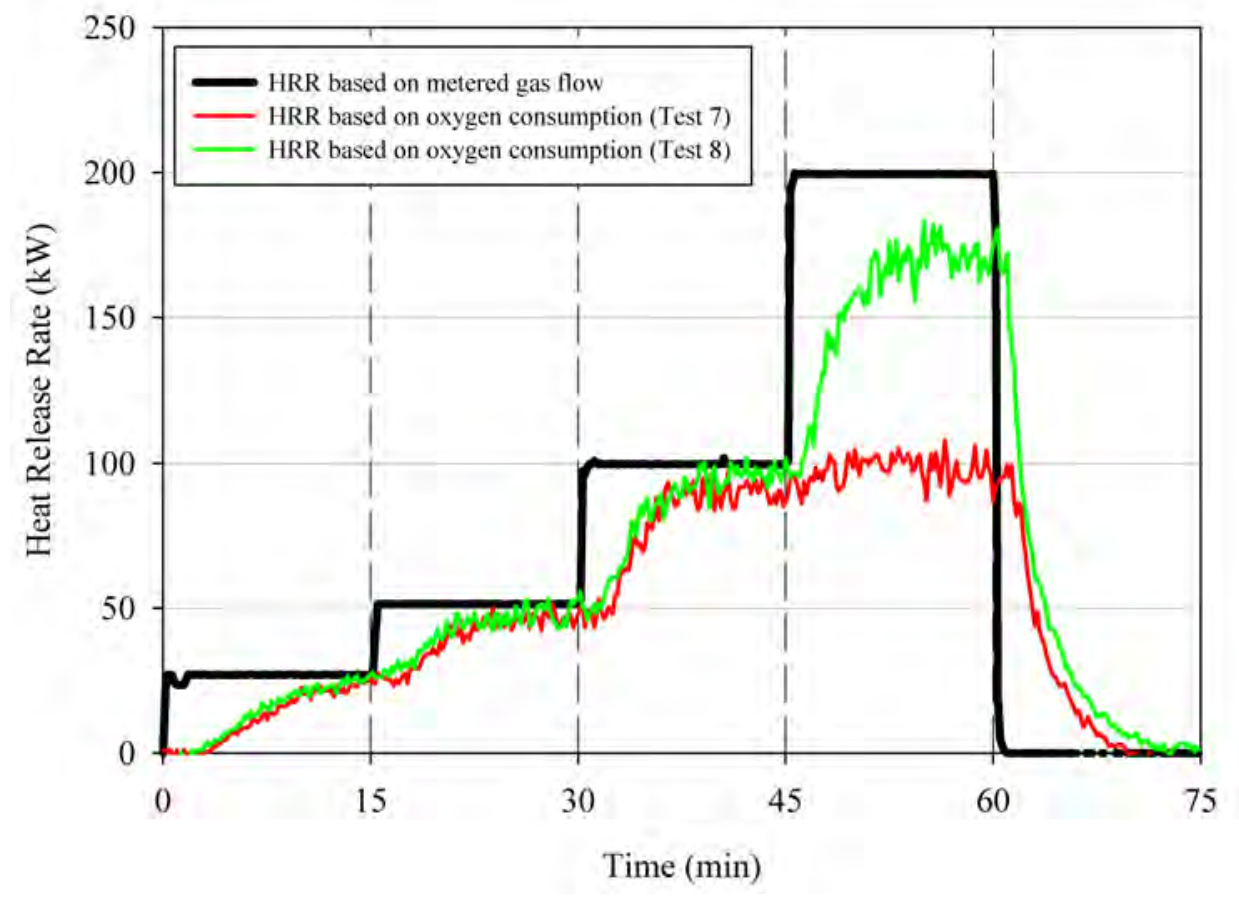

Fig. 31. Heat release rates, Test 7 and 8 . The black curve indicates the HRR based on the metered natural gas; the red and green are based on oxygen consumption calorimetry.

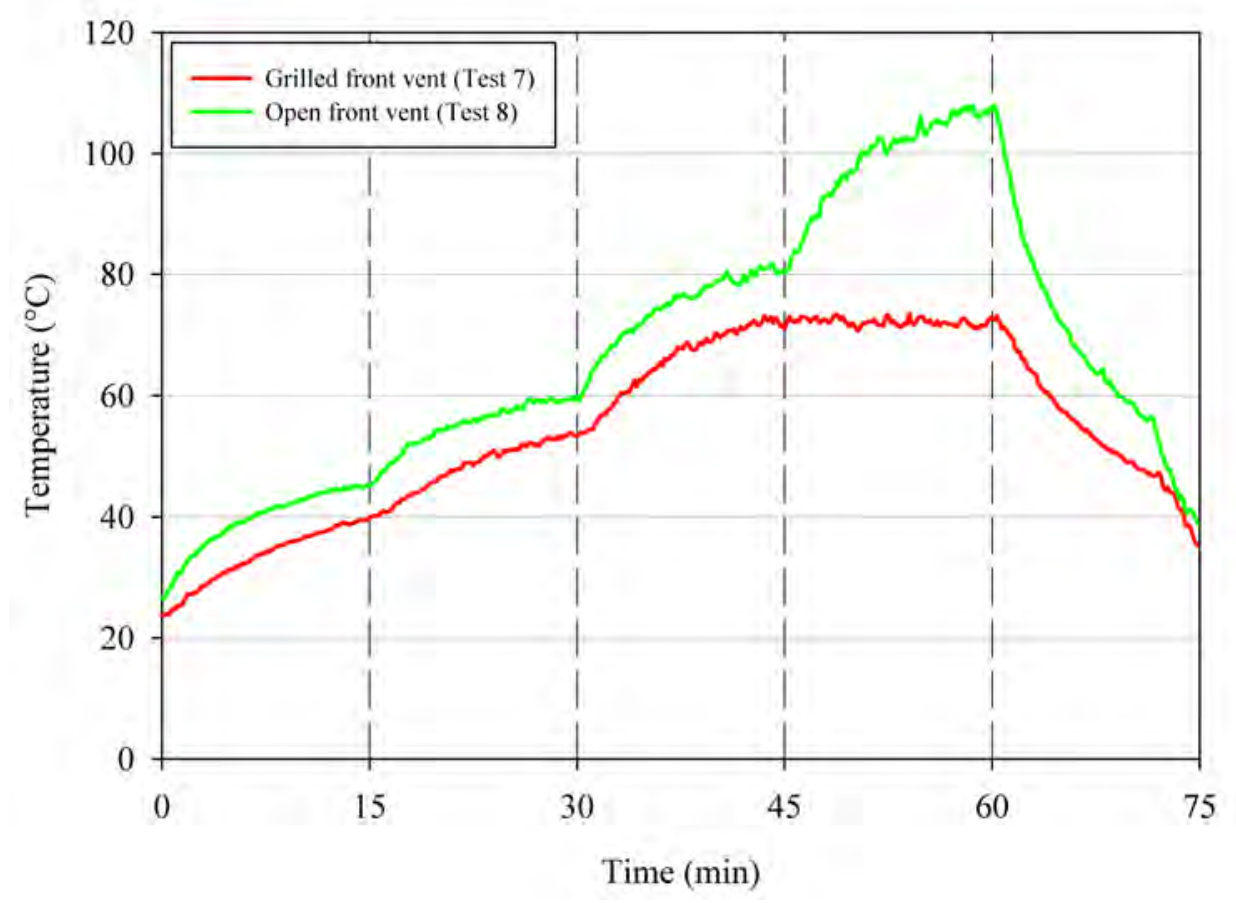

Fig. 32. HGL temperature, Tests 7 and 8. 


\subsubsection{Comparison with Experiments with no Cabinets}

Tests 11 and 12, conducted with no cabinet, were performed to test a hypothesis that the primary effect of a cabinet is to split the fire plume into two or more smaller plumes. Under this assumption, a zone model could account for the effect of a cabinet by simply splitting the fire into two or more smaller fires such that the total heat release rate is preserved. With this idea in mind, Tests 11 and 12 were performed using the same heat release rate sequence as Tests 1-6, the large cabinet experiments, but the two $30 \mathrm{~cm}$ square burners used in Tests 1-6 were separated by $90 \mathrm{~cm}(3 \mathrm{ft})$ and the cabinet was removed. Test 11 used the same HRR sequence as Tests 5-6 (open door), and Test 12 used the same sequence as Tests 1-4 (closed door).

Figures 33 and 34 show the hot gas layer temperature derived from the two vertical TC arrays along the compartment centerline. For the closed door experiments, Fig. 33, the highest recorded HGL temperature is for the case with no cabinet, followed in order by cases where the cabinet has less and less openings. This is expected, as the tighter cabinets trap more heat. For the open door experiments, Fig. 34, there is no obvious trend-the open cabinet plays less of a role in the overall temperature of the compartment. 


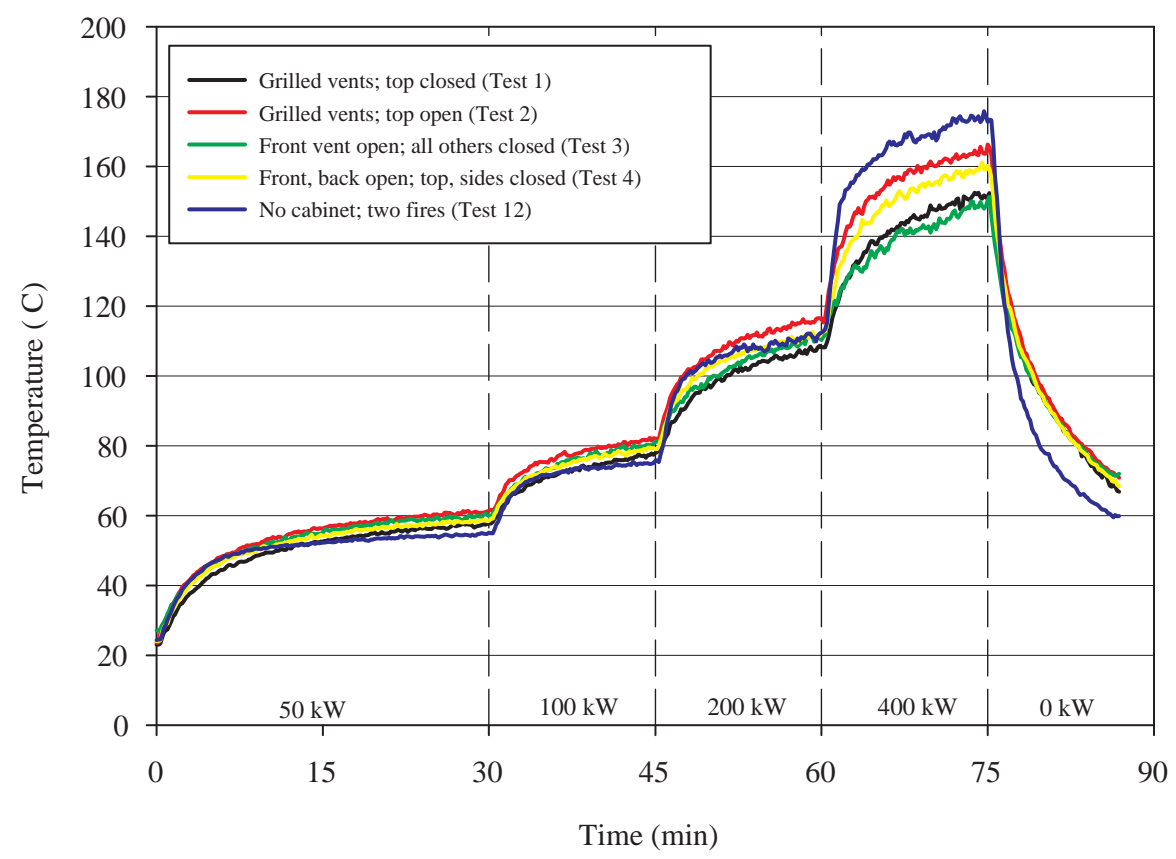

Fig. 33. HGL temperature for large cabinet experiments where front door is closed. The plot legends refer to the upper lateral and top vents.

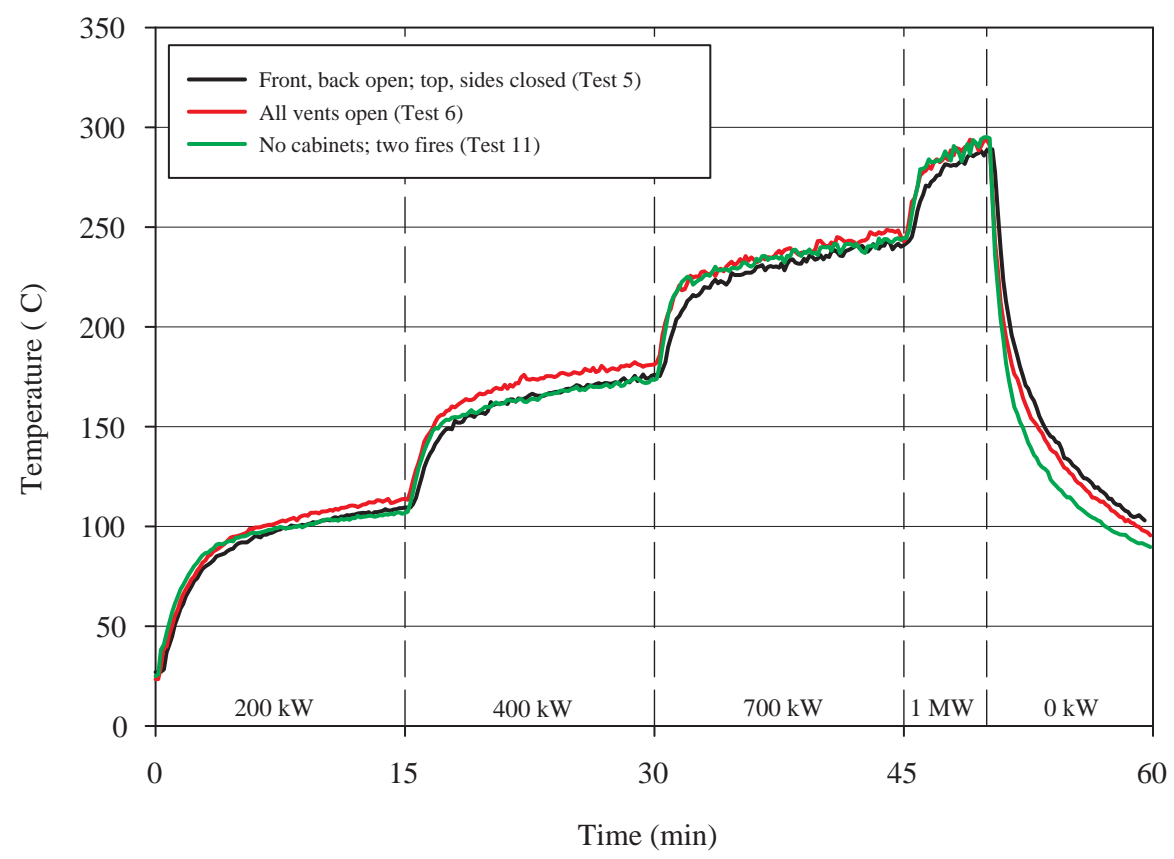

Fig. 34. HGL temperature for large cabinet experiments where front door is open. The plot legends refer to the upper lateral and top vents. 
Next, consider the effect that a cabinet has on the plume temperature. Figure 35 displays the measured plume temperatures at three heights above the burner. In Test 4, the cabinet is closed except for open vents near the top in the front and back, for which it can be assumed that half the fire's effluent exhausts through each one. In Test 12, there is no cabinet, and plume temperatures are recorded above one of the two $30 \mathrm{~cm}$ burners. Clearly, the spill plume from the upper cabinet vent in Test 4 is hotter than the unobstructed plume in Test 12. This observation does not support the hypothesis that these two fire scenarios can be taken as equivalent for modeling purposes. By trapping the heat from the fire, the cabinet effectively raises the origin of the obstructed spill plume.

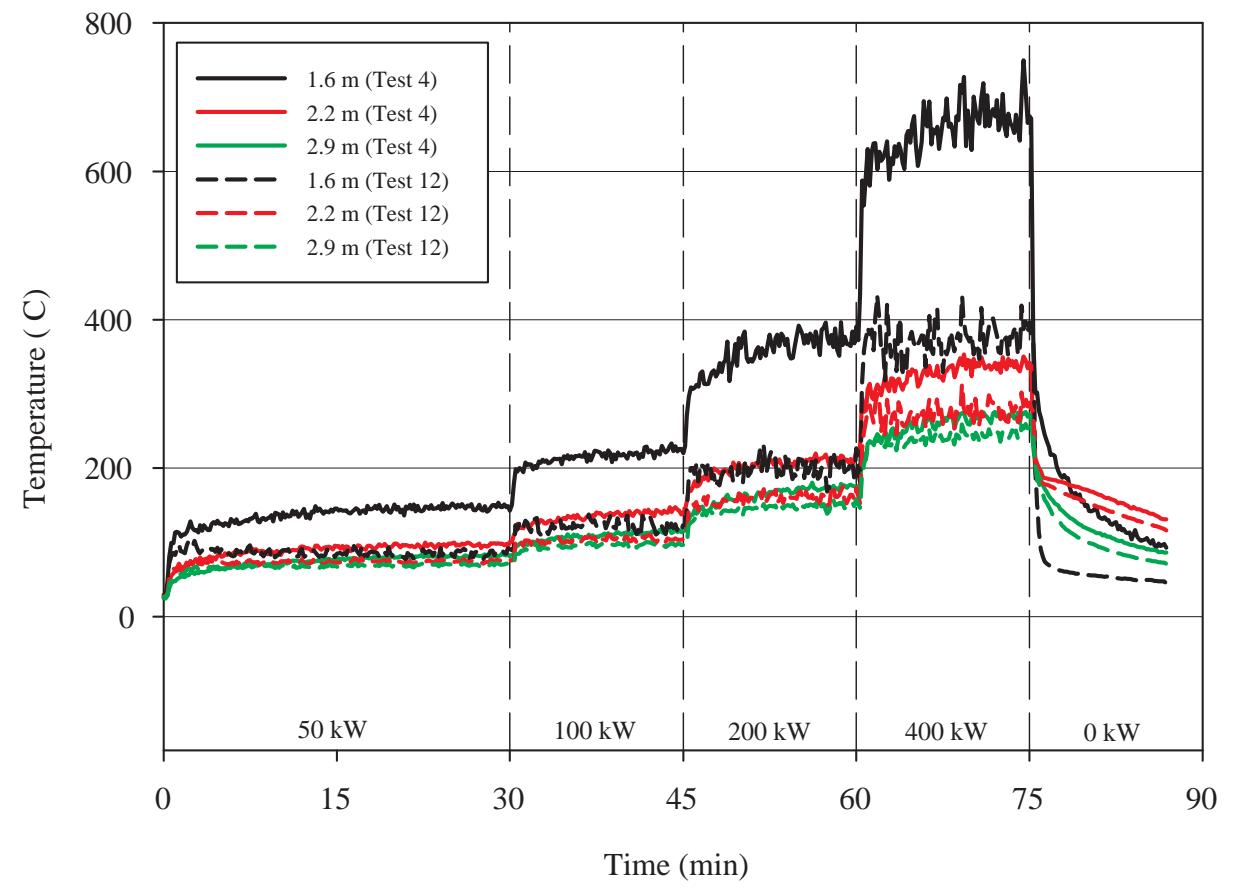

Fig. 35. Temperatures in the spill plume arising from one of two open vents in Test 4 and the plume above one of the two unobstructed single plumes in Test 12. 


\section{Conclusion}

This report documents two sets of fire experiments that investigate the effects of walls, corners, and small enclosures on a fire plume, its zone of influence, and the temperature and height of the hot gas layer within the compartment. The primary objective was to gather data for model validation, but some important trends were observed. These trends cannot be said to apply to all compartment and fire sizes; only the ones considered in these experiments.

1. While the corner had a noticeable effect on the flame height and plume temperatures, the wall did not. The plume temperatures and visible flame heights did not appear to change as the fire moved away from the wall.

2. The hot gas layer temperature and height were not noticeably affected by the location of the fire. This might be the result of the relatively large compartment size-smaller compartments might demonstrate a more noticeable effect.

The data for these experiments is stored in a repository used for FDS and CFAST validation: https://github.com/firemodels/exp/tree/master/NIST_NRC_Corner_Effects

\section{Acknowledgments}

Funding for this work was provided by the U.S. Nuclear Regulatory Commission, Office of Research, and by the Fire Risk Reduction in Buildings Program at the National Institute of Standards and Technology.

Matthew Bundy, Artur Chernovsky, and Laurean DeLauter of the National Fire Research Laboratory assisted in conducting these experiments and in processing the data. David Stroup of the U.S. Nuclear Regulatory Commission conducted infrared measurements of the cabinet experiments. 


\section{References}

[1] G. Heskestad. SFPE Handbook of Fire Protection Engineering, chapter Fire Plumes, Flame Height and Air Entrainment. Springer, New York, 5th edition, 2016.

[2] K. McGrattan and S. Bareham. Heat Release Rates of Electrical Enclosure Fires (HELEN-FIRE). NUREG/CR-7197, United States Nuclear Regulatory Commission, Washington, DC, 2015.

[3] A. Lindeman and M.H. Salley. Refining And Characterizing Heat Release Rates From Electrical Enclosures During Fire (RACHELLE-FIRE), Volume 1: Peak Heat Release Rates and Effect of Obstructed Plume. NUREG-2178, United States Nuclear Regulatory Commission, Washington, DC, April 2016.

[4] S. Welsh and P. Rubini. Three-dimensional Simulation of a Fire-Resistance Furnace. In Fire Safety Science - Proceedings of the Fifth International Symposium. International Association for Fire Safety Science, 1997.

[5] H. Ingason and U. Wickström. Measuring incident radiant heat flux using the plate thermometer. Fire Safety Journal, 42(2):161-166, 2007.

[6] A. Putorti, M. Melly, S. Bareham, and J. Praydis. Characterizing the Thermal Effects of High Energy Arc Faults. In 23rd International Conference on Structural Mechanics in Reactor Technology (SMiRT 23)_14th International Post-Conference Seminar on Fire Safety in Nuclear Power Plants and Installations, Salford, UK, August 2015.

[7] M.L. Janssens and H.C. Tran. Data Reduction of Room Tests for Zone Model Validation. Journal of Fire Science, 10:528-555, 1992.

[8] Y.P. He, A. Fernando, and M.C. Luo. Determination of interface height from measured parameter profile in enclosure fire experiment. Fire Safety Journal, 31:19-38, 1998.

[9] C.L. Beyler. SFPE Handbook of Fire Protection Engineering, chapter Fire Hazard Calculations for Large Open Hydrocarbon Fires. Springer, New York, 5th edition, 2016. 